\title{
Trilinear alternating forms on a vector space of dimension 7
}

\author{
Arjeh M. Cohen \& Aloysius G. Helminck \\ Centre for Mathematics and Computer Science \\ Kruislaan 413 \\ 1098 SJ Amsterdam \\ The Netherlands
}

ABSTRACT

For vector spaces of dimension at most 7 over fields of cohomological dimension at most 1 (including algebraically closed fields and finite fields) all trilinear alternating forms and their isotropy groups are determined.

\section{Introduction.}

Let $E$ be a vector space over the field $F$ of dimension $n<\infty$. Whereas the problem of classifying bilinear alternating forms on $E$ is well known and very elementary, the classification of trilinear alternating forms seems tractable for small values of $n$ only. By the classification of $r$-linear alternating forms on $E$ we mean the determination of equivalence classes of these forms with respect to the following equivalence relation:

Two $r$-linear forms $f, f^{\prime}$ on $E$ are said to be equivalent if there is a linear transformation $g \in G L(E)$, the general linear group on $E$, such that

$$
f\left(x_{1}, \ldots, x_{r}\right)=f^{\prime}\left(g x_{1}, \ldots, g x_{r}\right) \quad\left(x_{1}, \ldots, x_{r} \in E\right) .
$$

Letting $g \in G L(E)$ act on the vector space of $r$-linear forms $f$ on $E$ by means of

$$
(g \cdot f)\left(x_{1}, \ldots, x_{r}\right)=f\left(g^{-1} x_{1}, \ldots, g^{-1} x_{r}\right) \quad\left(x_{1}, \ldots, x_{r} \in E\right),
$$

we can rewrite (1) as $g \cdot f=f^{\prime}$. We shall often write $g f$ instead of $g \cdot f$. The equivalence classes are the $G L(E)$-orbits under this action. An $r$-linear form $f$ on $E$ is called alternating if for all $x_{1}, \ldots, x_{r} \in E$ with $x_{i}=x_{j}$ for at least two distinct $i, j$ $(1 \leqslant i, j \leqslant r)$, we have $f\left(x_{1}, \ldots, x_{r}\right)=0$. The vector space of all $r$-linear alternating forms on $E$ will be denoted by $\operatorname{Alt}_{r}(E)$. The classification of $r$-linear alternating forms on $E$ consists of the determination of all $G L(E)$-orbits in Alt $_{r}(E)$. We shall write $G=G L(E)$, and $G_{f}=\{g \in G \mid g f=f\}$ for any $f \in$ Alt $_{r}(E)$; we shall refer to 
$G_{f}$ as the stabilizer (in $G$ ) of $f$. Obviously, $G_{f}$ is an algebraic group defined over $F$; in fact, it is a Zariski closed subgroup of $G$. If $K$ is an extension field of $F$, then any $r$-linear form $f$ on $E$ has a unique extension to an $r$-linear form $f^{K}$ on $E \otimes_{F} K$. Two $r$-linear forms $f, f^{\prime}$ on $E$ are called $K$-equivalent whenever their extensions $f^{K}$, $\left(f^{\prime}\right)^{K}$ are equivalent. We shall denote by $\bar{F}$ the algebraic closure of $F$ and we shall write $\bar{E}=E \otimes_{F} \bar{F}, \bar{G}=G L(\bar{E})$, and $\bar{f}=f^{\bar{F}}$.

Now, $\operatorname{dim} \bar{G}$ and $\operatorname{dim} \bar{G}_{f}$ are well-defined integers and the orbit $\overline{G f}$ of $\bar{f}$ has the structure of an algebraic variety of dimension $\operatorname{dim} \bar{G}-\operatorname{dim}(\overline{G f})$, see BorEL [1]. Since $\operatorname{dim} \bar{G}=n^{2}$ and $\operatorname{dim} \operatorname{Alt}_{r}(\bar{E})=\left(\begin{array}{l}n \\ r\end{array}\right)$, this yields

$$
\operatorname{dim} \bar{G}_{f} \geqslant n^{2}-\left(\begin{array}{l}
n \\
r
\end{array}\right) .
$$

If $\bar{G}$ has finitely many orbits in $\operatorname{Alt}_{r}(\bar{E})$, there must be a form $\bar{f}$ in $\operatorname{Alt}_{r}(\bar{E})$ for which equality holds in (3). But equality implies $n^{2} \geqslant\left(\begin{array}{l}n \\ r\end{array}\right)$. Hence, if Alt ${ }_{r}(\bar{E})$ consists of finitely many equivalence classes, either $r \leqslant 2$, or $r=3$ and $n \leqslant 8$. This illustrates why the problem of classifying trilinear alternating forms differs from its bilinear counterpart.

If $r=2$, there are finitely many $G$-orbits, see DieudonNE [4]. We shall see in the sequel that for arbitrary $F$ this is no longer true if $r=3$ and $n=7$. However, if $F$ is algebraically closed, $r=3$ and $n \leqslant 7$, there are finitely many $G$-orbits. The classification in this case was carried out by J.A. SCHOUTEN [7] in 1931 for $F=\mathbb{C}$, and, independently, by CRESP [3] in 1976 for $F$ algebraically closed of characteristic $\neq 2,3$. GUREVICH [5] gives an answer to the classification problem with $F=\mathbb{C}$, $r=3$ and $n=8$. In this paper, we present a relatively short proof of the classification for $F$ algebraically closed of arbitrary characteristic, $r=3$ and $n=7$. Furthermore, we compute the stabilizers in $G$ of representatives of each of its orbits and derive the classification for $n \leqslant 7$ and $r=3$ over certain non-algebraically closed fields - including all finite fields - by use of (noncommutative) first order Galois cohomology. In particular for a finite field $F$, it turns out that the number of projective $G L(n, F)$-orbits on $\wedge^{3} F^{n}$ is 4 if $n=6$, and 11 if $n=7$.

After finishing this work, it has come to our knowledge that MIGLIORE [10] has also studied trilinear alternating forms on $F^{7}$ for finite fields $F$.

The outline of the paper is as follows. The main results are stated in Section 2. In the hope that for finite fields this classification will be of some relevance to finite geometry and combinatorics, we have included two corollaries of this classification. Section 2 ends with an elementary but basic lemma for the classification over an algebraically closed field. The classification over these fields appears in Section 4, while Section 3 is devoted to the computation of the stabilizers of the forms given in Section 2. Finally, in Section 5 the necessary Galois cohomology is introduced and an account is given of the classification over a field of cohomological dimension at most 1 . 


\section{Notation and Statement of Results.}

In this section, we let $E=F^{n}$ be the standard vector space over the field $F$ of dimension $n$, consisting of column vectors, and denote by $e_{1}, \ldots, e_{n}$ the standard basis vectors. The dual $E^{\star}$ of $E$ will be interpreted as a vector space of row vectors. Thus, $e_{1}^{\top}, \ldots, e_{n}^{\top}$, where $x^{\top}$ is the transposed of $x \in E$, is a dual basis of $e_{1}, \ldots, e_{n}$, i.e., is a basis of $E^{*}$ with

$$
e_{i}^{\top} e_{j}= \begin{cases}1 & \text { if } i=j \\ 0 & \text { otherwise. }\end{cases}
$$

By well-known theory, cf. BourbaKI [2], the space Alt ${ }_{r}(E)$ may be identified with the skew-symmetric tensor product $\wedge^{r} E^{*}$ of $r$ copies of $E^{*}$. In fact, we shall identify the two by viewing $x_{1}^{\top} \wedge x_{2}^{\top} \wedge \ldots x_{r}^{\top}$ for $x_{1}, \ldots, x_{r} \in E$ as elements of $\operatorname{Alt}_{r}(E)$ by means of

$$
x_{1}^{\top} \wedge \cdots \wedge x_{r}^{\top}\left(u_{1}, \ldots, u_{r}\right)=\sum_{\sigma \in S_{r}} s g(\sigma) \prod_{i=1}^{r} x_{i}^{\top} u_{\sigma(i)}\left(u_{1}, \ldots, u_{r} \in E\right)
$$

where $S_{r}$ stands for the symmetric group on $r$ symbols and $s g(\sigma)$ is the sign of $\sigma \in S_{r}$. Obviously,

$$
\left\{e_{i_{1}}^{\top} \wedge \cdots \wedge e_{i_{r}}^{\top} \mid 1 \leqslant i_{1}<i_{2}<\ldots<i_{r} \leqslant n\right\}
$$

is a basis of $\operatorname{Alt}_{r}(E)$.

The action of $g \in G=G L(E)$ on $\operatorname{Alt}_{r}(E)$ defined in (2) is determined by

$$
g\left(x_{1}^{\top} \wedge \cdots \wedge x_{r}^{\top}\right)=x_{1}^{\top} g^{-1} \wedge \cdots \wedge x_{r}^{\top} g^{-1} \quad\left(x_{1}, \ldots, x_{r} \in E\right),
$$

where $x^{\top} h$ for $x \in E$ and $h \in G$ is the element of $E^{*}$ satisfying $\left(x^{\top} h\right) v=x^{\top}(h v)$ for all $v \in E$. In order to simplify notation, we shall often abbreviate $e_{i}^{\top}$ to $\underline{i}$. Also, we shall write $\underline{i j}$ for $\underline{i} \wedge \dot{j}$ and $i j k$ for $\underline{i} \wedge \underline{j} \wedge \underline{k}$, where $1 \leqslant i, j, k \leqslant n$. We are now ready to formulate the main results.

2.1. Theorem. Let $E$ be a vector space of dimension 7 over an algebraically closed field $F$. Then any nonzero trilinear alternating form on $E$ is equivalent to exactly one of the nine forms $f_{i}$ for $i=1, \ldots, 9$ of Table 1 . The stabilizer $G_{i}$ of $f_{i}$ in $G=G L(E)$ has the structure given in Table 1 .

Here, $F_{\star}^{m}$, for $m \in \mathbb{N}$, denotes a group with composition series of length $m$ all of whose $m$ factors are isomorphic to the additive group of the field $F, F^{*}$ denotes the multiplicative group of $F$, and $\mu_{3}$ the subgroup $\left\{x \in F \mid x^{3}=1\right\}$. Furthermore, $\lambda, \mu$ are such that

$$
P_{\lambda}(x)= \begin{cases}X^{2}-\lambda & \text { if } F \text { has odd characteristic } \\ X^{2}+\lambda X+1 & \text { otherwise }\end{cases}
$$

and $P_{\mu}(X)=X^{3}-\mu$ are irreducible in $F[X]$. We note that Table 1 contains only a rough description of the isomorphism type of $G_{f}$. A full description can be found in Section 3. 
Table 1

Alternating trilinear forms on a vector space of dimension 7 over a perfect field $F$.

\begin{tabular}{|c|c|c|}
\hline name & form & description of $G_{f}$ \\
\hline $\begin{array}{l}f_{1} \\
f_{2} \\
f_{3} \\
f_{4} \\
f_{5} \\
f_{6} \\
f_{7} \\
f_{8} \\
f_{9} \\
f_{10, \lambda} \\
\\
f_{11, \lambda} \\
f_{12, \mu}\end{array}$ & 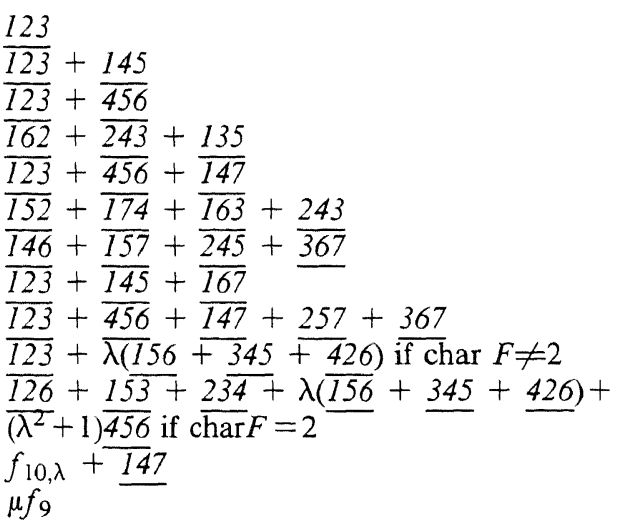 & $\begin{array}{l}F_{\star}^{12} \cdot(S L(3, F) \cdot G L(4, F)) \\
F_{\star}^{14} \cdot\left(G L(2, F) \cdot\left(S p(4, F) \cdot F^{\star}\right)\right) \\
\left(F_{*}^{6} \cdot\left(S L(3, F) \cdot S L(3, F) \cdot F^{\star}\right)\right) \cdot \mathbb{Z}_{2} \\
F_{\star}^{14} \cdot\left(G L(3, F) \cdot F^{\star}\right) \\
\left(F_{\star}^{10} \cdot(G L(2, F) \cdot G L(2, F))\right) \cdot \mathbb{Z}_{2} \\
F_{\star}^{12} \cdot\left(S L(3, F) \cdot F^{\star}\right) \\
F_{\star}^{8} \cdot\left((G L(2, F) \cdot G L(2, F)) / F^{\star}\right) \\
F_{\star}^{6} \cdot\left(S p(6, F) \cdot F^{\star}\right) \\
G_{2}(F) \cdot \mu_{3} \\
\left(F_{*}^{6} \cdot\left(F^{\star} \cdot S L(3, K)\right)\right) \cdot \mathbb{Z}_{2} \\
\left(F_{\star}^{10} G L(2, K)\right) \cdot \mathbb{Z}_{2} \\
G_{2}(F) \cdot \mu_{3}\end{array}$ \\
\hline
\end{tabular}

A form $f \in \operatorname{Alt}_{3}(E)$, where $E=F^{n}$, can conveniently be represented by a diagram in the following way. A diagram consists of vertices and lines which are labelled 3cycles of vertices. The vertices are the coordinates $1, \ldots, n$. A line is a 3 -cycle $(i, j, k)$ with $\lambda=f\left(e_{i}, e_{j}, e_{k}\right) \neq 0$ whose label is $\lambda$. In a picture of a diagram the lines $(i, j, k)$ for which $i<j<k$ does not hold are suppressed. If $f\left(e_{i}, e_{j}, e_{k}\right)=1$, the label is not drawn at all. See Figure 1 for an example.

A perfect field is said to have cohomological dimension at most 1 if the Galois group of its algebraic closure has cohomological dimension at most 1; cf. SERRE [8], where it is also shown that finite fields have cohomological dimension at most 1.

2.2. Theorem. If $E$ is a vector space of dimension 7 over a perfect field $F$ of cohomological dimension at most 1 , then any nonzero trilinear form is equivalent to one of the forms $f_{i}(1 \leqslant i \leqslant 9), f_{j, \lambda}(j=10,11), f_{12, \mu}$ of Table 1 . Moreover, the only pairs of equivalent forms in Table 1 occur among the pairs $f_{j, \alpha}, f_{j, \beta}$, where either $j=10,11$ and $P_{\alpha}(X), P_{\beta}(X)$ define isomorphic extension fields of $F$ in the respective cases, or $j=12$ and the polynomials $X^{3}-\alpha, X^{3}-\beta$ define isomorphic cubic extension fields of $F(\sqrt[3]{-1})$ and $\alpha^{-1} \beta$ has a cube root in $F(\sqrt[3]{-1})$. 
Table 2

The number of forms in $\mathrm{Alt}_{3}\left(F^{7}\right), F$ a finite field of order $q$, equivalent to one of the forms in Table 1.

\begin{tabular}{|l|l|l|}
\hline form & \multicolumn{1}{|c|}{$\left|G / G_{f_{1}}\right|$} & degree \\
\hline \hline$f_{1}$ & $\left(q^{7}-1\right)\left(q^{5}-1\right)\left(q^{3}+1\right) /(q-1)^{2}$ & 13 \\
$f_{2}$ & $\left(q^{7}-1\right)\left(q^{5}-1\right)\left(q^{4}+q^{2}+1\right)\left(q^{2}+q+1\right) q^{2}$ & 20 \\
$f_{3}$ & $\frac{1}{2}\left(q^{7}-1\right)\left(q^{5}-1\right)\left(q^{3}+1\right)\left(q^{2}+1\right) q^{9}$ & 26 \\
$f_{4}$ & $\left(q^{7}-1\right)\left(q^{6}-1\right)\left(q^{5}-1\right)\left(q^{3}+q^{2}+q+1\right) q^{4}$ & 25 \\
$f_{5}$ & $\frac{1}{2}\left(q^{7}-1\right)\left(q^{6}-1\right)\left(q^{5}-1\right)\left(q^{2}+1\right)\left(q^{2}+q+1\right) q^{9}$ & 31 \\
$f_{6}$ & $\left(q^{7}-1\right)\left(q^{6}-1\right)\left(q^{5}-1\right)\left(q^{4}-1\right) q^{6}$ & 28 \\
$f_{7}$ & $\left(q^{7}-1\right)\left(q^{6}-1\right)\left(q^{5}-1\right)\left(q^{3}-1\right)\left(q^{2}+1\right) q^{11}$ & 34 \\
$f_{8}$ & $\left(q^{7}-1\right)\left(q^{5}-1\right)\left(q^{3}-1\right) q^{6}$ & 21 \\
$f_{9}$ & $\epsilon\left(q^{7}-1\right)\left(q^{5}-1\right)\left(q^{4}-1\right)\left(q^{3}-1\right)(q-1) q^{15}$ & 35 \\
$f_{10, \lambda}$ & $\frac{1}{2}\left(q^{7}-1\right)\left(q^{5}-1\right)\left(q^{3}-1\right)\left(q^{2}-1\right) q^{9}$ & 26 \\
$f_{11, \lambda}$ & $\frac{1}{2}\left(q^{7}-1\right)\left(q^{6}-1\right)\left(q^{5}-1\right)\left(q^{3}-1\right)(q-1) q^{9}$ & 31 \\
$f_{12, \mu}$ & $\frac{1-\epsilon}{2}\left(q^{7}-1\right)\left(q^{5}-1\right)\left(q^{4}-1\right)\left(q^{3}-1\right)(q-1) q^{15}$ & 35 \\
$f_{12, \mu^{2}}$ & $\frac{1-\epsilon}{2}\left(q^{7}-1\right)\left(q^{5}-1\right)\left(q^{4}-1\right)\left(q^{3}-1\right)(q-1) q^{15}$ & 35 \\
\hline
\end{tabular}

2.3. Corollary. Let $E, F$ be as in Theorem 2.2. If $H$ is a subgroup of $G L(E)$ which is irreducible on $E$ and fixes a nonzero trilinear alternating form on $E$, then $H$ is a subgroup of the Chevalley group of type $G_{2}$ over $F$ extended by $\mu_{3}=\left\{x \in F^{*} \mid x^{3}=1\right\}$.

Proof. By inspection of the stabilizers of Table 1 and their action on $E$ (see Section 3 ), it is immediate that $G_{f_{9}}, G_{f_{12, \mu}}$ are the only stabilizers of trilinear alternating forms which are irreducible on $E$. Since $H$ is irreducible and fixes a nonzero element of $\operatorname{Alt}_{3}(E)$, it must be a subgroup of a conjugate of $G_{f_{9}}, G_{f_{12, \mu}}$, and hence of $G_{2}(F) \mu_{3}$.

2.4. Corollary. Let $F$ be a finite field of order $q$. Then the number of forms in Alt $_{3}(E)$, with $E=F^{7}$, equivalent to one of the forms in Table 1 is as given in Table 2.

Proof. Given $f \in \operatorname{Alt}_{3}(E)$, the number in question is $|G| /\left|G_{f}\right|$, where $G=G L(E)$ has order $q^{21} \prod_{i=1}^{7}\left(q^{i}-1\right)$. Since $G_{f}$ is conjugate to $G_{f_{i}}$ if $f$ is equivalent to $f_{i}$, and the right hand side is known by Table 1 , the result follows by straightforward computation.

Here, $\epsilon=1$ if $\operatorname{gcd}(q-1,3)=1$ and $\epsilon=\frac{1}{3}$ otherwise; $\lambda, \mu$ are as in Table 1. (Observe that if $\operatorname{gcd}(q-1,3)=1$, then $f_{12, \mu}$ and $f_{12, \mu^{2}}$ do not occur.)

We finish this section by introducing the basic tools of the proof of the above theorems. Let $E, F, G$ be as before and $r \geqslant 1$. For $f \in$ Alt $_{r}(E)$ we define the kernel 


$$
f_{3}^{a}=a_{3} \underline{12}-a_{2} \underline{13}+a_{1} \underline{23}+a_{6} \underline{45}-a_{5} \underline{46}+a_{4} \underline{56} .
$$

It readily follows that $R_{0}\left(f_{3}\right)=\left\langle e_{7}\right\rangle$ and that

$$
R_{\leqslant 1}\left(f_{3}\right)=\left\langle e_{1}, e_{2}, e_{3}, e_{7}\right\rangle \cup\left\langle e_{4}, e_{5}, e_{6}, e_{7}\right\rangle \text {. }
$$

Suppose $g \in G_{3}$. Then $g$ either stabilizes each of the two linear subspaces whose union is $R \leqslant 1\left(f_{2}\right)$, or interchanges them. Moreover, $g$ stabilizes $\left\langle e_{7}\right\rangle$. Hence, after multiplication of $g$ by an element of $A$, we may assume that $g$ is of diagonal form with entries $(\lambda, 1,1, \mu, 1,1,1)$ for some $\lambda, \mu \in F^{*}$. As in 3.1, it follows that $\lambda=\mu=1$, so that $g \in A$.

3.4. The form $f_{4}=\underline{162}+\underline{243}+\underline{135}$ has stabilizer $G_{4}=A$, where

$$
A=\left\{\begin{array}{ccc}
h & 0_{3 \times 3} & 0_{3 \times 1} \\
r & h\left(\operatorname{det} h^{-1}\right) & 0_{3 \times 1} \\
a & \lambda
\end{array} \mid \begin{array}{c}
h \in G L(3, F), \lambda \in F^{*} \\
a \in F^{1 \times 6}, r \in F^{3 \times 3} \\
\text { trace } r=0
\end{array}\right\},
$$

so $G_{4} \cong F_{*}^{14}:\left(G L(3, F) \times F^{\star}\right)$.

Proof. A simple computation shows that $R_{0}(f)=\left\langle e_{7}\right\rangle$ and that $R_{1}(f)=\left\langle e_{4}, e_{5}, e_{6}\right\rangle \backslash\{0\}$. Suppose $g \in G$. Then $g$ stabilizes $R_{0}(f)$ and $R_{1}(f) \cup\{0\}$, so after multiplication by an element of $A$, we may restrict attention to $g$ of the form

$$
g=\left(\begin{array}{ccc}
a & 0_{3 \times 4} \\
\alpha & 0 & 0 \\
0 & \\
0_{3 \times 3} & 1_{4}
\end{array}\right)
$$

for some $\alpha \in F$ and $a=\left(a_{i j}\right) \in G L(3, F)$. Now,

$$
\begin{aligned}
g^{-1} f_{4}= & \left(a_{11} a_{22}-a_{12} a_{21}\right) \underline{162}+\left(a_{11} a_{23}-a_{13} a_{21}\right) \underline{163}+ \\
& \left(a_{12} a_{23}-a_{13} a_{22}\right) \underline{263}+\alpha\left(a_{23} a_{32}-a_{22} a_{33}\right) \underline{123}+ \\
& \left(a_{22} a_{31}-a_{21} a_{32}\right) \underline{124}+\left(a_{23} a_{31}-a_{21} a_{33}\right) \underline{134}+ \\
& \left(a_{23} a_{32}-a_{22} a_{33}\right) \underline{234}+\left(a_{11} a_{33}-a_{13} a_{31}\right) \underline{135}+ \\
& \left(a_{12} a_{33}-a_{13} a_{22}\right) \underline{235},
\end{aligned}
$$

by straightforward use of (5), so that $g \cdot f_{4}=f_{4}$ implies $\alpha=0$ and $a= \pm I_{3}$. It follows that $g \in A$. 
3.5. The form $f_{5}=\underline{123}+\underline{456}+\underline{147}$ has stabilizer $G_{5}=A$, where

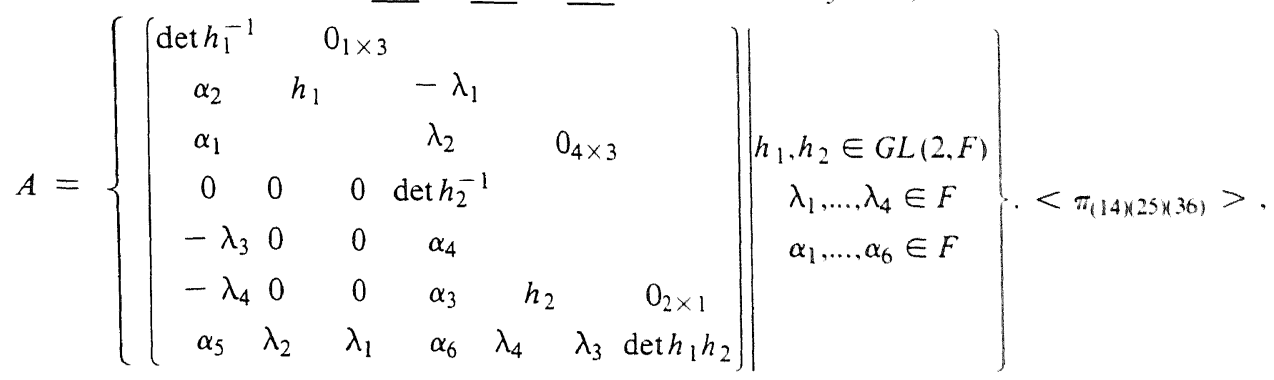
so $G_{5} \cong\left(F_{\star}^{10}:(G L(2, F) \times G L(2, F))\right) \cdot \mathbb{Z}_{2}$.

Proof. By computation, $R \leqslant 1\left(f_{5}\right)=\left\langle e_{5}, e_{6}, e_{7}\right\rangle \cup\left\langle e_{2}, e_{3}, e_{7}\right\rangle$ and $\left.R \leqslant 2\left(f_{5}\right)=\left\langle e_{1}, e_{2}, e_{3}, e_{5}, e_{6}, e_{7}\right\rangle \cup<e_{2}, \ldots, e_{7}\right\rangle$. Suppose $g \in G_{5}$. Then either $g$ stabilizes each of the two 3-dimensional subspaces in $R \leqslant 2\left(f_{5}\right)$. or interchanges them. Hence $g$ stabilizes their linear $\operatorname{span}\left\langle e_{2}, e_{3}, e_{5}, e_{6}, e_{7}\right\rangle$ as well as their intersection $\left\langle e_{7}\right\rangle$. After multiplication of $g$ by a suitable element of $A$, we may, therefore, assume that $g$ stabilizes $\left\langle e_{1}, e_{4}\right\rangle$. But then $g$ either interchanges $\left\langle e_{1}\right\rangle,\left\langle e_{4}\right\rangle$ or stabilizes each of them (as it stabilizes $\left.\left.R \leqslant 2\left(f_{5}\right) \cap<e_{1}, e_{4}\right\rangle\right)$. In the former case, multiply by $\pi_{(14 \times 25)(36)} \in A$, so as to reduce study to the case where $g\left\langle e_{i}\right\rangle=\left\langle e_{i}\right\rangle$ for each $i(i=1,4)$. In fact, by multiplication with a suitable diagonal element of $A$, we may even assume $g e_{l}=e_{l}$ for $i=1,4$. Since $R \leqslant 1\left(f_{5}\right) \cap \operatorname{ker} f_{5}^{e_{1}}=\left\langle e_{5}, e_{6}\right\rangle$ and $R \leqslant 1\left(f_{5}\right) \cap \operatorname{ker} f_{5}^{t^{4}}=$ $\left\langle e_{2}, e_{3}\right\rangle$, these subspaces must be $g$-invariant. It readily follows that $g$ belongs to $A$.

3.6. The form $f_{6}=\underline{152}+\underline{174}+\underline{163}+\underline{243}$ has stabilizer $G_{6}=A$, where $A=A_{1} A_{2}$ with

$$
\left.\begin{array}{c}
A_{1}=\left\{\left(\begin{array}{ccccccc}
\lambda_{1}^{-1} & 0 & 0 & 0 & 0 & 0 & 0 \\
\lambda_{2} & 1 & 0 & 0 & 0 & 0 & 0 \\
\lambda_{3} & 0 & 1 & 0 & 0 & 0 & 0 \\
\lambda_{4} & 0 & 0 & 1 & 0 & 0 & 0 \\
\lambda_{5} & 0 & \lambda_{4} & \lambda_{3} & \lambda_{1} & 0 & 0 \\
\lambda_{6} & 0 & 0 & \lambda_{2} & 0 & \lambda_{1} & 0 \\
\lambda_{7} & 0 & 0 & 0 & 0 & 0 & \lambda_{1}
\end{array}\right) \mid \begin{array}{c} 
\\
\lambda_{1} \in F^{*} \\
\lambda_{2} \ldots, \lambda_{7} \in F
\end{array}\right\} \\
A_{2}=\left\{\left(\begin{array}{ccc}
1 & 0 & 0 \\
0 & a & 0 \\
0 & h & \left(a^{\top}\right)^{-1}
\end{array}\right) \mid h \in F^{3 \times 3}, a \in \operatorname{SL(3,F)}\right. \\
h=h^{\top}
\end{array}\right\},
$$

so $G_{6} \cong F_{\star}^{12}:\left(S L(3, F) \times F^{*}\right)$. 
Proof. Notice that $R \leqslant 1\left(f_{6}\right)=<e_{5}, e_{6}, e_{7}>$ and $\left.R \leqslant 2\left(f_{6}\right)=<e_{2}, \ldots, e_{7}\right\rangle$. Suppose $g \in G_{6}$. After multiplication of $g$ by an element of $A_{1}$ from the left, we may assume that $g e_{1}=e_{1}$. Since $g$ stabilizes $\left\langle e_{5}, e_{6}, e_{7}\right\rangle$, after multiplication of $g$ by an element of $A_{2}$, we may restrict attention to the case where $g$ has the form

$$
\left(\begin{array}{cccccccc}
1 & 0 & 0 & 0 & & & \\
0 & & & & & & \\
0 & & a & & & 0_{4 \times 3} & \\
0 & & & & & & \\
0 & 0 & \alpha_{4} & \alpha_{3} & \alpha_{1} & 0 & 0 \\
0 & 0 & 0 & \alpha_{2} & 0 & 1 & 0 \\
0 & 0 & 0 & 0 & 0 & 0 & 1
\end{array}\right)
$$

for some $a \in G L(3, F)$, with $\alpha_{1} \in F^{\star}$, and $\alpha_{2}, \alpha_{3}, \alpha_{4} \in F$. By a computation using (5), we obtain

$$
\begin{aligned}
f_{6}=g^{-1} f_{6}= & \alpha_{1} \underline{15}\left(2 a^{\top}\right)+\alpha_{3} \underline{14}\left(\underline{2} a^{\top}\right)+\alpha_{4} \underline{13}\left(2 a^{\top}\right)+ \\
& \left.\underline{17} \underline{4} a^{\top}\right)+\underline{16}\left(\underline{3} a^{\top}\right)+\alpha_{2} \underline{14}\left(\underline{3} a^{\top}\right)+ \\
& (\operatorname{det} a) \underline{243} .
\end{aligned}
$$

It follows that $\underline{3} a^{\top}=\underline{3}, \underline{4} a^{\top}=\underline{4}$ and $\underline{2} a^{\top}=\alpha_{1}^{-1} \underline{2}$, so that

$$
a=\left(\begin{array}{rrr}
\alpha_{1}^{-1} & 0 & 0 \\
0 & 1 & 0 \\
0 & 0 & 1
\end{array}\right) \text {. }
$$

Hence,

$$
\underline{243}=f_{6}-\underline{152}-\underline{174}-\underline{163}=\underline{14}\left(\alpha_{1}^{-1} \alpha_{3} \underline{2}+\alpha_{2} \underline{3}\right)+\underline{13}\left(\alpha_{4} \underline{2}\right)+(\operatorname{det} a) \underline{243} .
$$

This implies $\alpha_{1}=(\operatorname{det} a)^{-1}=1$ and $\alpha_{2}=\alpha_{3}=\alpha_{4}=0$, so that $g=1 \in A$, indeed.

3.7. The form $f_{7}=\underline{146}+\underline{157}+\underline{245}+\underline{367}$ has stabilizer $G_{7}=A$ where $A=A_{1} A_{2}$ with

$$
A_{1}=\left\{\left(\left\{\begin{array}{ccccc} 
& \alpha_{1} & \beta_{1} & \beta_{3} & -\alpha_{3} \\
1_{3} & \alpha_{2} & \beta_{2} & \beta_{1} & -\alpha_{1} \\
& \alpha_{3} & \beta_{2} & \gamma_{3} & \delta_{3} \\
0_{4 \times 3} & & & 1_{4}
\end{array}\right] \mid \alpha_{1}, \alpha_{2}, \alpha_{3}, \beta_{1}, \beta_{2}, \beta_{3}, \gamma_{3}, \delta_{3} \in F\right\}\right. \text {, and }
$$




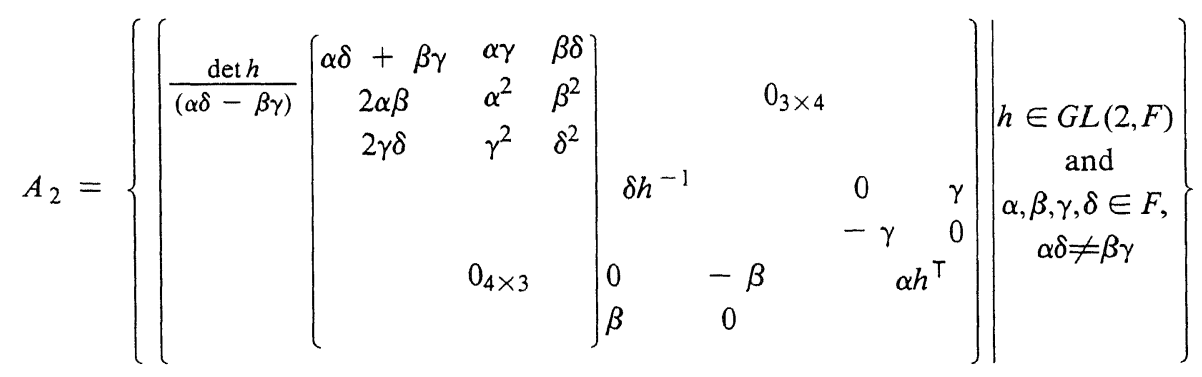

so $G_{7} \cong F_{\star}^{8}:\left((G L(2, F) \times G L(2, F)) / F^{\star}\right)$.

Proof. A direct computation leads to the relations

$$
\begin{aligned}
& R_{\leqslant 1}\left(f_{7}\right)=\left\{x_{1} e_{1}+x_{2} e_{2}+x_{3} e_{3} \mid x_{1}^{2}=x_{2} x_{3}\right\}, \text { and } \\
& R_{\leqslant 2}\left(f_{7}\right)=\left\{\sum_{i=1}^{7} x_{i} e_{i} \mid x_{4} x_{6}+x_{5} x_{7}=0\right\} .
\end{aligned}
$$

Suppose $g \in G_{7}$. Since $\left\langle e_{1}, e_{2}, e_{3}\right\rangle$ is the linear subspace of $E$ spanned by $R \leqslant 2\left(f_{7}\right)$, it is left invariant by $g$. Write $W_{0}=\left\langle e_{4}, e_{5}, e_{6}, e_{7}\right\rangle$.

Let $W$ be a 4-dimensional subspace of $E$ with $W \cap\left\langle e_{1}, e_{2}, e_{3}\right\rangle=\{0\}$ and $f_{7}(W, W, W)=0$. Then there is an element $r \in G$ of the form

$$
\left[\begin{array}{ll}
1_{3} & a \\
0_{3 \times 4} & 1_{4}
\end{array}\right] \text { with } a=\left(a_{i j}\right) \in F^{3 \times 4},
$$

such that $W=r W_{0}$. The requirement $f_{7}(W, W, W)=0$ implies

$$
\left\{\begin{array}{l}
0=g^{-1} f_{7}\left(e_{4}, e_{5}, e_{6}\right)=-a_{12}+a_{23} \\
0=g^{-1} f_{7}\left(e_{4}, e_{5}, e_{7}\right)=a_{11}+a_{24} \\
0=g^{-1} f_{7}\left(e_{4}, e_{6}, e_{7}\right)=a_{31}+a_{14} \\
0=g^{-1} f_{7}\left(e_{5}, e_{6}, e_{7}\right)=a_{32}-a_{13} .
\end{array} .\right.
$$

Hence, $r \in A_{1}$. Thus, up to multiplication of $g$ by an element of $A_{1}$, we may assume that $g$ stabilizes $W_{0}$. Consider the group $H$ generated by the elements of $A_{2}$ restricted to $W_{0}$. This is a subgroup of index 2 in the full subgroup of the linear group on $W_{0}$ preserving the quadric

$$
\left\{\sum_{i=4}^{7} x_{i} e_{i} \mid x_{4} x_{6}+x_{5} x_{7}=0\right\},
$$

see Dieudonne [4]. In fact, $H$ is isomorphic to $(G L(2, F) \times G L(2, F)) / F^{*}$, the central product of two copies of $G L(2, F)$. Multiplication of $g$ by a suitable element of $A_{2}$, therefore leads to the case where we have either $g \mid w_{0}=1$ or 
$g \mid w_{0}=\pi_{(46)}$. However, $\pi_{(46)}$ cannot be extended to a linear transformation on $E$ preserving $f_{7}$ (i.e., to a member of $G_{7}$ ), for, denoting such an extension by $h$ and writing $h e_{2}=x_{1} e_{1}+x_{2} e_{2}+x_{3} e_{3}$, we would then have

$$
\mathrm{l}=h^{-1} f\left(e_{2}, e_{4}, e_{5}\right)=f\left(h e_{2}, e_{6}, e_{5}\right)=\sum_{i=1}^{3} x_{i} f\left(e_{i}, e_{6}, e_{5}\right)=0,
$$

which is absurd. The conclusion is that we are left with the case where $g \mid w_{0}=1$. Explicit verification of $f_{7}=g^{-1} f_{7}$ by means of (5), yields that $g=1$, so that $g \in A$.

3.8. The form $f_{8}=\underline{123}+\underline{145}+\underline{167}$ has stabilizer $G_{8}=A$ with $A=A_{1} A_{2}$, where

$$
\begin{aligned}
& A_{1}=\left\{\left(\begin{array}{ccccccc}
\lambda^{-1} & & & & & & \\
& 0 & 0 & 0 & 0 & 0 & 0 \\
& \lambda & 0 & 0 & 0 & 0 & 0 \\
a & 0 & 1 & 0 & 0 & 0 & 0 \\
& 0 & 0 & \lambda & 0 & 0 & 0 \\
0 & 0 & 0 & 1 & 0 & 0 \\
0 & 0 & 0 & 0 & \lambda & 0 \\
0 & 0 & 0 & 0 & 0 & 1
\end{array}\right) \mid a \in F^{6} \times 1, \lambda \in F^{*}\right\} \text {, and } \\
& A_{2}=\left\{\left(\begin{array}{ll}
1 & 0_{1 \times 6} \\
0_{1 \times 6} & h
\end{array}\right) \mid \begin{array}{l}
h \in G L(6, F) \\
h \text { preserves } 23 \\
23
\end{array} \underline{45}+\underline{67}\right\} .
\end{aligned}
$$

Thus $G_{8} \cong F^{6}:\left(S p(6, F) \cdot F^{\star}\right)$.

Proof. Observe that $R \leqslant 1\left(f_{8}\right)=\left\langle e_{2}, \ldots, e_{7}\right\rangle$. Let $g \in G_{8}$. Since $g$ stabilizes $R_{1}\left(f_{8}\right)$, we may assume that $G$ fixes $e_{1}$ (after multiplication by a suitable element of $A_{1}$ ). But then $g$ fixes $f_{8}^{\ell_{1}}$, whence $g \in A_{2}$, so $g \in A$.

3.9. The form $f_{9}=\underline{123}+\underline{456}+\underline{147}+\underline{257}+\underline{367}$ has stabilizer $G_{9}=A$, with $A=G_{2}(F) \mu_{3}$, where $\overline{G_{2}}(F) \overline{\text { is }}$ the Chevalley group of type $G_{2}$ over $F$, and $\mu_{3}=\left\{\alpha \in F \mid \alpha^{3}=1\right\}$.

Proof. Direct computation shows

$$
R_{\leqslant 2}\left(f_{9}\right)=R_{2}\left(f_{9}\right) \cup\{0\}=\left\{\sum_{i=1}^{7} x_{i} e_{i} \in E \mid x_{7}^{2}=x_{1} x_{4}+x_{2} x_{5}+x_{3} x_{6}\right\} .
$$

Let $H$ denote the full subgroup of $G$ preserving the quadric $R_{2}\left(f_{9}\right)$ and set $H_{1}=H \cap S L(E)$. Then $H_{1}$ preserves the quadratic form

$$
Q(x)=-x_{7}^{2}+x_{1} x_{4}+x_{2} x_{5}+x_{3} x_{6} \quad\left(x=\left(x_{1}, \ldots, x_{7}\right) \in E\right)
$$

and each $h \in H$ is of the form $\lambda h_{1}$ for some $\lambda \in F^{\star}$ and some $h_{1} \in H_{1}$. More- 
over, $G_{2}(F)$ is the subgroup of $H_{1}$ preserving $f_{9}$, cf. SPRINGER [9]. In fact, one can show that the bilinear multiplication on $E$ defined by $f_{9}(x, y, z)=B(x \cdot y, z)$ for all $x, y, z \in E$, where $B$ is the bilinear form associated with $Q$, is the restriction to the orthoplement of unity in the 8-dimensional split algebra of the octonions. In order to see this, take the 8-dimensional split algebra of the octaves to be the set of matrices of the form

$$
\left[\begin{array}{ll}
\alpha & v \\
w & \beta
\end{array}\right] \text { where } \alpha, \beta \in F \text { and } v, w \in F^{3}
$$

supplied with the usual matrix addition, with multiplication

$$
\left[\begin{array}{ll}
\alpha & v \\
w & \beta
\end{array}\right]\left[\begin{array}{ll}
\alpha^{\prime} & v^{\prime} \\
w^{\prime} & \beta^{\prime}
\end{array}\right]=\left(\begin{array}{cc}
\alpha \alpha^{\prime}-v^{\prime} w^{\prime} & \alpha v^{\prime}+\beta^{\prime} v+w \times w^{\prime} \\
\alpha^{\prime} w+\beta w^{\prime}+v \times v^{\prime} & \beta \beta^{\prime}-v^{\prime} \cdot w
\end{array}\right)
$$

where - and $\times$ stand for the standard inner and outer product on $F^{3}$, respectively, and with quadratic form $Q$ given by

$$
Q\left(\left[\begin{array}{ll}
\alpha & v \\
w & \beta
\end{array}\right]\right)=\alpha \beta+v \cdot w .
$$

The unity of this algebra is $\left(\begin{array}{ll}1 & 0 \\ 0 & 1\end{array}\right)$; its orthoplement is spanned by the basis

$$
e_{1}=\left(\begin{array}{cc}
1 & 0 \\
0 & -1
\end{array}\right), e_{i+1}=\left(\begin{array}{ll}
0 & \epsilon_{i} \\
0 & 0
\end{array}\right), e_{i+4}=\left(\begin{array}{ll}
0 & 0 \\
\epsilon_{i} & 0
\end{array}\right)
$$

where $i=1,2,3$ and $\left(\epsilon_{i}\right)_{i}$ is the standard basis of $F^{3}$. A straightforward computation shows that the restrictions of $Q$ and $(x, y, z) \mapsto B(x \cdot y, z)$ to the orthoplement of the unity are as indicated above when taken with respect to $e_{1}, \ldots, e_{7}$.

We need two properties of the group $A$ in order to finish the proof. They are formulated in the following lemma.

Lemma. (i) The group $G_{2}(F)$, viewed as subgroup of $G$ preserving $f_{9}$ and $B$ is transitive on the set of unordered pairs of mutually non-orthogonal (with respect to B) totally isotropic 1-dimensional subspaces of $E$.

(ii) If $g \in G$ and $\mu \in F^{*}$ satisfy $g f_{9}=\mu f_{9}$, then there is $\lambda \in F^{*}$ with $\mu=\lambda^{-3}$ and $g \in \lambda A$.

Statement (i) is a well-known property of $G_{2}(F)$, see for instance SPRINGER [9].

Before proving part (ii) of the lemma, let us show how it leads to $G_{9}=A$. Suppose $g \in G_{9}$. Write $g=\lambda h$ with $\lambda \in F^{\star}$ and $h \in H_{1}$. Then $f_{9}=g f_{9}=\lambda^{-3}\left(h f_{9}\right)$, so $h f_{9}=\lambda^{3} f_{9}$. By part (ii) of the lemma, we have $h \in \mu_{3} \lambda^{-1} A=\lambda^{-1} A$, whence $g=\lambda h \in A$, as wanted.

It remains to prove part (ii) of the lemma. To this end, let $g, \mu$ be as in the hypothesis. Notice that $g$ preserves $R_{2}\left(f_{9}\right)$, the quadric defined by $Q$. By part (i) of the lemma, we may assume that $g\left\langle e_{1}\right\rangle=\left\langle e_{1}\right\rangle$ and $g\left\langle e_{4}\right\rangle=\left\langle e_{4}\right\rangle$ (after multiplication of $g$ by a suitable member of $A$ ). Thus, $g$ preserves $e_{1}^{\perp}$ and 
$e_{4}^{\frac{1}{4}}$, where $\perp$ is taken with respect to $B$. The following subspaces of $E$ are preserved by $g: R_{0}\left(f_{9}^{\ell_{1}}\right) \cap e_{1}^{\perp} \cap e_{4}^{\frac{1}{4}}=\left\langle e_{5}, e_{6}\right\rangle, R_{0}\left(f_{9}^{e_{4}}\right) \cap e_{1}^{\perp} \cap e_{4}^{\frac{1}{4}}=$ $\left.<e_{2}, e_{3}\right\rangle$, and $\left(R_{0}\left(f_{9}^{e_{1}}\right)+R_{0}\left(f_{9}^{e_{4}}\right)\right)^{\perp}=\left\langle e_{7}\right\rangle$. Therefore, $g$ must have the form

$$
\left[\begin{array}{ccccc}
\alpha_{1} & & & \\
& a & & & \\
& & & & \\
& & & & \\
& & b & \\
& & & \alpha_{7}
\end{array}\right] \quad \begin{aligned}
& \text { where } \alpha_{1}, \alpha_{4}, \alpha_{7} \in F^{*} \\
& \text { and } a, b \in G L(2, F) .
\end{aligned}
$$

Since

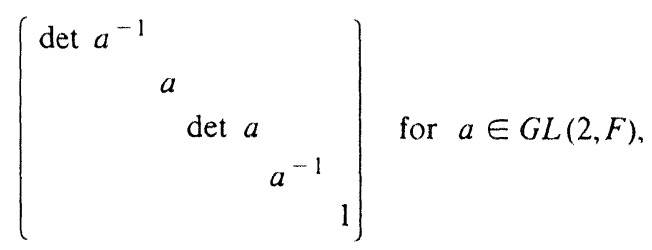

represents an element of $G_{2}(F)$, we may restrict attention to the case where $g e_{2}=e_{2}$ and $g e_{3}=e_{3}$. But then $g$ preserves $e_{2}^{\frac{1}{2}} \cap\left\langle e_{5}, e_{6}\right\rangle=\left\langle e_{6}\right\rangle$ and $e_{4}^{\frac{1}{1}} \cap\left\langle e_{5}, e_{6}\right\rangle=\left\langle e_{5}\right\rangle$, so $g$ has diagonal form $\left(\alpha_{1}, 1,1, \alpha_{4}, \alpha_{5}, \alpha_{6}, \alpha_{7}\right)$. From $g f_{9}=\mu f_{9}$, we get $\mu^{-1}=\alpha_{1}=\alpha_{4} \alpha_{5} \alpha_{6}=\alpha_{1} \alpha_{4} \alpha_{7}=\alpha_{5} \alpha_{7}=\alpha_{6} \alpha_{7}$, whence $\alpha_{1}=\mu^{-1}, \alpha_{4}^{3}=\mu, \alpha_{5}=\alpha_{6}=\alpha_{4} \mu^{-1}, \alpha_{7}=\alpha_{4}^{-1}$. Set $\lambda=\alpha_{4}^{-1}$. Then $\mu=\lambda^{3}$ and $g$ has diagonal form $\left(\lambda^{3}, 1,1, \lambda^{-1}, \lambda^{2}, \lambda^{2}, \lambda\right)=\lambda\left(\lambda^{2}, \lambda^{-1}, \lambda^{-1}, \lambda^{-2}, \lambda, \lambda, 1\right)$, whence $g \in \lambda A$.

3.10. Fix $\lambda \in F$ such that the polynomial

$$
P_{\lambda}(X)=\left\{\begin{array}{ll}
X^{2}-\lambda & \text { if } F \text { has odd characterictic } \\
X^{2}+\lambda X+1 & \text { if } F \text { has even characteristic }
\end{array}\right. \text {, }
$$

is irreducible, and set $K=F(\alpha)$, where $\alpha$ is a root of $P_{\lambda}(X)$. Then, the form

$$
f_{10, \lambda}=\left\{\begin{array}{l}
\frac{123}{126}+\lambda(\underline{156}+\underline{345}+\underline{153}+\underline{426}) \text { if } F \text { has odd characteristic, } \\
(\underline{156}+\underline{345}+\underline{426})+\left(\lambda^{2}+1\right) \underline{456} \text { otherwise }
\end{array}\right.
$$

is $K$-equivalent but not equivalent to $f_{3}$. Furthermore, let $\sigma$ denote the generator of the Galois group of $K$ over $F$, and set

$$
g=\left(\begin{array}{ccc}
1_{3} & -\alpha l_{3} & 0_{3 \times 1} \\
1_{3} & \alpha l_{3} & 0_{3 \times 1} \\
0_{1 \times 3} & 0_{1 \times 3} & \frac{1}{4}
\end{array}\right) \quad \text { if } F \text { has odd characteristic, }
$$




$$
g=\left(\begin{array}{ccccccc}
\alpha & 0 & 0 & 1 & 0 & 0 & 0 \\
0 & \alpha+\lambda & 0 & 0 & \alpha \lambda+\lambda^{2}+1 & 0 & 0 \\
0 & 0 & \lambda^{-1} & 0 & 0 & \alpha \lambda^{-1}+1 & 0 \\
\alpha+\lambda & 0 & 0 & 1 & 0 & 0 & 0 \\
0 & \alpha & 0 & 0 & \alpha \lambda+1 & 0 & 0 \\
0 & 0 & \lambda^{-1} & 0 & 0 & \alpha \lambda^{-1} & 0 \\
0 & 0 & 0 & 0 & 0 & 0 & \lambda^{-1}
\end{array}\right) \text { otherwise }
$$

The stabilizer of $f_{10, \lambda}$ is $G_{10, \lambda}=g^{-1} \mathrm{Ag}$,

$$
\text { where } \left.A=\left\{\begin{array}{lll}
k & 0_{3 \times 3} & 0_{6 \times 1} \\
0_{3 \times 3} & k^{\sigma} & \\
a & a^{\sigma} & \rho
\end{array}\right) \mid \begin{array}{l}
k \in S L(3, K) \\
a \in K^{3} \\
\rho \in F^{*}
\end{array}\right\}<\pi_{(14)(25)(36)}>\text {. }
$$

In particular, $G_{10, \lambda} \cong\left(F_{\star}^{6}:\left(F^{*} \times S L(3, K)\right)\right) \cdot \mathbb{Z}_{2}$.

Proof. Since

$$
g \cdot f_{10, \lambda}^{K}= \begin{cases}4 f_{3}^{K} & \text { if } F \text { has odd characteristic, } \\ f_{3}^{K} & \text { otherwise, }\end{cases}
$$

$f_{10, \lambda}$ is $K$-equivalent to $f_{3}$. Put $G_{3}^{K}=G_{f_{3}^{k}}^{K}$. For $h \in G_{3}^{K}$, its conjugate $g^{-1} h g$ belongs to $G$ if and only if it is fixed by $\sigma$, i.e., if and only if

$$
h^{\sigma}=\left(g^{\sigma} g^{-1}\right) h\left(g^{\sigma} g^{-1}\right)^{-1} \text {. }
$$

But $g^{\sigma} g^{-1}=\left(\begin{array}{ccc}0_{3 \times 3} & 1_{3} & \\ 1_{3} & 0_{3 \times 3} & 0_{6 \times 1} \\ 0_{1 \times 6} & 1\end{array}\right)$, so by use of 3.3 , we readily

find that the elements $h \in G_{3}^{K}$ satisfying (8) are contained in $A$. The conclusion is that any $x \in G_{10, \lambda}$ is of the form $x=g^{-1} h g$ with $h \in A$, so that $G_{10, \lambda}=g^{-1} A g$.

It may be worthy of note that the restriction of $f_{10, \lambda}$ to $\left\langle e_{1}, \ldots, e_{6}\right\rangle$ can be obtained as the restriction to $\left\langle e_{1}, e_{2}, e_{3}\right\rangle \otimes K$ (viewed as a vector space over $F$ ) of trace $_{K / F}\left(f_{1}^{K}\right)$.

3.11. Let $\lambda, P_{\lambda}(X), K=F(\alpha), \sigma, g$ be as in 3.10. The form $f_{11, \lambda}=f_{10, \lambda}+147$ is $K$-equivalent but not equivalent to $f_{5}$. The stabilizer of $f_{11, \lambda}$ is $G_{11, \lambda}=g^{-T_{A g}}$, where 


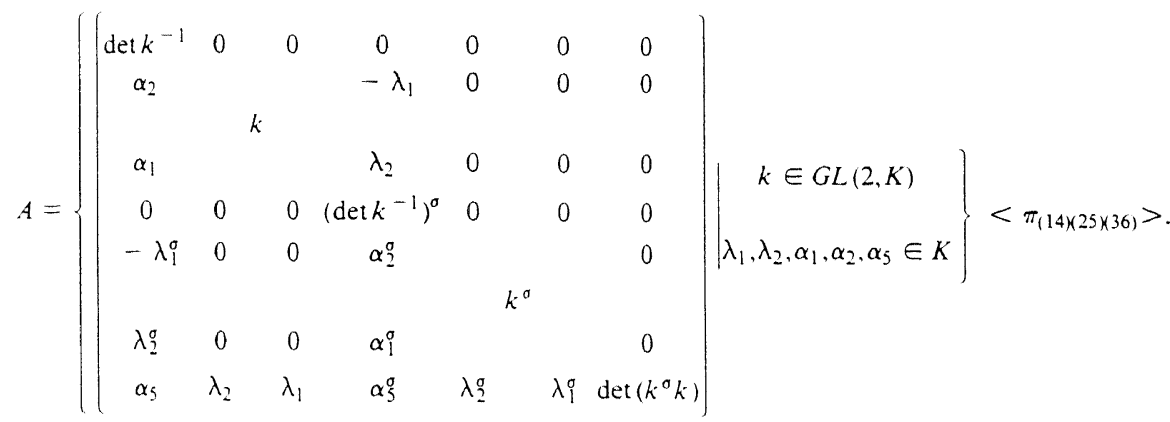

In particular, $G_{11, \lambda} \cong\left(F_{\star}^{10} . G L(2, K)\right) \cdot \mathbb{Z}_{2}$.

Proof. Since $g \cdot f_{11, \lambda}=\mu f_{5}$, with $\mu=4$ if $F$ has even characteristic and $\mu=1$ otherwise, $f_{11, \lambda}$ is $K$-equivalent to $f_{5}$.

Put $G_{5}^{K}=G_{f_{s}^{K}}^{K}$, and recall that this group has been described in $\S 3.5$. For

$$
h=\left(\begin{array}{ccccccc}
\operatorname{det} g_{1}^{-1} & 0 & 0 & 0 & 0 & 0 & 0 \\
\alpha_{2} & & & -\lambda_{1} & 0 & 0 & 0 \\
& & k & & & & \\
\alpha_{1} & & & \lambda_{2} & 0 & 0 & 0 \\
0 & 0 & 0 & \operatorname{det} g_{2}^{-1} & 0 & 0 & 0 \\
-\lambda_{1}^{\sigma} & 0 & 0 & \alpha_{4} & & & 0 \\
\lambda_{2}^{\sigma} & 0 & 0 & \alpha_{5} & & & 0 \\
\alpha_{5} & \lambda_{2} & \lambda_{1} & \alpha_{6} & \lambda_{4} & \lambda_{3} & \operatorname{det}\left(g_{1} g_{2}\right)
\end{array}\right) \in G_{5}^{K},
$$

with $\alpha_{1}, \alpha_{2}, \alpha_{3}, \alpha_{4}, \alpha_{5}, \quad \alpha_{6}, \lambda_{1}, \lambda_{2}, \lambda_{3}, \quad \lambda_{4} \in K \quad$ and $g_{1}, g_{2} \in G L(2, K)$, we have $g^{-1} h g \in G_{11, \lambda}$ if and only if $h^{\sigma} \in G$, i.e., if and only if $h \in A$. This settles 3.11.

3.12. Suppose $K=F(\alpha)$ is a cubic field extension of $F$ with $\alpha^{3}=\mu$ for some $\mu \in F^{\star}$. Then $f_{12, \mu}=\mu f_{9}$ and $f_{12, \mu^{2}}=\mu^{2} f_{9}$ are mutually inequivalent forms, both inequivalent but $K$-equivalent to $f_{9}$, and the stabilizer $G_{9}$ of $f_{9}$ coincides with both the stabilizer $G_{12, \mu}$ of $f_{12, \mu}$ and $G_{12, \mu^{2}}$.

Proof. This is immediate from the lemma in 3.9.

\section{The Classification over an Algebraically Closed Field.}

In this section, we prove Theorem 2.1. Thus, we assume that $F=\bar{F}$ is an algebraically closed field, and set $E=F^{7}$.

The following result will be used frequently. 
4.1. Lemma. Suppose $D$ is a linear subspace of $E$ of dimension $m \geqslant 3$. If $f \in \operatorname{Alt}_{3}(D)$ and $e_{1}, e_{2}, e_{3} \in D$ satisfy $f\left(e_{1}, e_{2}, e_{3}\right) \neq 0$, then $e_{1}, e_{2}, e_{3}$ can be extended to a basis $e_{1}, \ldots, e_{m}$ of $D$ such that

$$
f\left(e_{1}, e_{2}, e_{3}\right) \neq 0 \text { and } f\left(e_{i}, e_{j}, e_{k}\right)=0 \text { for } 1 \leqslant i \leqslant j \leqslant 3 \text { and } 4 \leqslant k \leqslant m \text {. }
$$

Proof. The intersection of the kernels of the three linear functionals $x \mapsto f\left(e_{i}, e_{j}, x\right)$ for $1 \leqslant i<j \leqslant 3$ is a linear subspace of $D$ of dimension $m-3$. Taking $e_{4}, \ldots, e_{m}$ to be a basis of this subspace, we obtain $e_{1}, \ldots, e_{m}$ as stated.

Notice that the relations in (9) are invariant under linear transformations preserving the subspaces $\left\langle e_{1}, e_{2}, e_{3}\right\rangle$ and $\left\langle e_{4}, \ldots, e_{m}\right\rangle$. Let $f \in \operatorname{Alt}_{3}(E)$. Write $C=\operatorname{ker} f$ and let $D$ be a complement of $C$ in $E$, so that $E=C \oplus D$. Let $m$ be the dimension of $D$. Now, $G=G L(E)$ is transitive on ordered pairs of subspaces of given dimensions forming a decomposition of $E$. Hence, for any basis $e_{1}, \ldots, e_{7}$, we can replace $f$ by an equivalent form whose kernel is $C=\left\langle e_{m+1}, \ldots, e_{7}\right\rangle$ (complemented by $D=\left\langle e_{1}, \ldots, e_{m}\right\rangle$ ).

From now on, let $f \in \mathrm{Alt}_{3}(E)$.

4.2. Lemma. Suppose $f \neq 0$. Then $\mathrm{rk} f \geqslant 3$. If, moreover, $\operatorname{rk} f \leqslant 4$, then $\operatorname{rk} f=3$ and $f$ is equivalent to $f_{1}$.

Proof. Set $r=\mathrm{rk} f$, and let $D$ be a complement of ker $f$ with basis $e_{1}, \ldots, e_{r}$. If $r<3$, then $f \mid D=0$, so $f=0$, a contradiction. Hence $r \geqslant 3$. If $r \leqslant 4$, then $f \mid D$ is equivalent to $f_{1} \mid D$, whence $f$ is equivalent to $f_{1}$ by Lemma 4.1.

4.3. Lemma. If $\mathrm{rk} f=5$, then $f$ is equivalent to $f_{2}$.

Proof. Let $e_{1}, \ldots, e_{5}$ be a basis of a complement $D$ of ker $f$ such that (9) is satisfied for $\left.f\right|_{D}$. Since $\operatorname{rk} f=5$, one of the values $f\left(e_{i}, e_{4}, e_{5}\right)$, for $i=1,2,3$, must be nonzero. Without loss of generality, we may assume $f\left(e_{1}, e_{4}, e_{5}\right)=1$. Thus,

$$
f=\underline{123}+\underline{145}+\underline{\lambda 245}+\underline{\mu 345} \text { for some } \lambda, \mu \in F \text {. }
$$

Taking $g \in G$ with $g \underline{1}=\underline{1}-\lambda \underline{2}-\mu \underline{3}$ and $g \underline{i}=\underline{i}$ for $i>1$, we get $g f=f_{3}$, whence the lemma.

4.4. Lemma. Suppose $\lambda \in F^{\star}, \mu \in F$, and let

$$
\begin{aligned}
& h_{\lambda, \mu}=\underline{123}+\underline{146}+\underline{356}+\lambda \underline{456}+\mu \underline{236}, \text { and } \\
& h_{\lambda}=\underline{123}+\underline{146}+\underline{456} .
\end{aligned}
$$

Then $h_{\lambda, \mu}$ and $h_{\lambda}$ are both equivalent to $f_{3}$.

Proof. Take $g \in G$ with $g \cdot \underline{4}=\underline{4}-\lambda^{-1} \underline{3}, g \cdot \underline{2}=\underline{2}-\left(\lambda^{-1}-\mu\right) \underline{6}, g \cdot \underline{i}=\underline{i}$ for $i=1,3,5,6,7$. Then $g \cdot h_{\lambda, \mu}=\bar{h}_{\lambda}$. It therefore remains to prove that $\bar{h}_{\lambda}$ is equivalent to $f_{3}$. Take $g_{1} \in G$ with $g_{1} \cdot \underline{5}=\underline{5}-\lambda^{-1} \underline{l}$ and $g_{1} \cdot \underline{i}=\underline{i}$ for $i \neq 5$. Then $g_{1} \cdot h_{\lambda}=\underline{123}+\lambda \underline{456}$, which is clearly equivalent to $f_{3}$. 


\section{Figure 1.}

The diagrams of the forms $h_{\lambda, \mu}$ and $h_{\lambda}$.
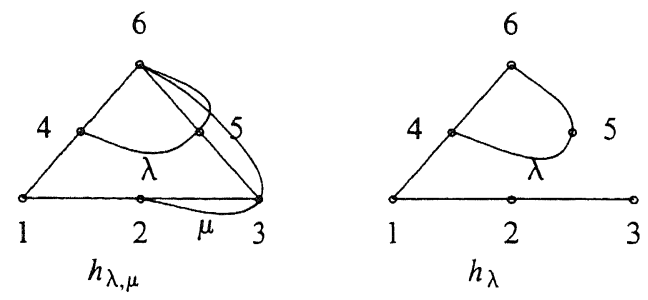

4.5. Lemma. For $\lambda, \mu \in F$, suppose $f=145+246+\underline{356}+\mu 123+\lambda 456$. Then $f$ is equivalent to $f_{4}$ whenever $\mu=0$ or $\lambda^{2} \mu=-4$, and equivalent to $\overline{f_{3} \text { otherwise. }}$

Proof. If $\mu=0$, it is readily checked that $f$ is equivalent to $f_{4}$. Thus, let $\mu \neq 0$. There is $\chi \in F^{*}$ with $\chi^{2}=\mu$ (since $F$ is algebraically closed). Now, $h \in G$, determined by $h \cdot \underline{4}=\chi \underline{4}, h \cdot \underline{i}=\chi^{-1} \underline{i}$ for $i=1,2, h \underline{j}=j$ for $j=3,5,6,7$ applied to $f$ yields $h \cdot f=\overline{145}+24 \overline{6}+356 \overline{+} 123+\lambda \chi 456$. Therefore, without loss of generality, we assume that $\mu=1$. Let $\alpha, \overline{\beta, \gamma}, \delta, \zeta, \overline{\eta \in F}$ satisfy $\alpha \beta, \gamma \delta, \zeta \eta \neq 1$, and take $g \in G$ with $g \cdot \underline{7}=\underline{7}$ and

$$
\begin{aligned}
& g \cdot 1=\underline{1}+\alpha \underline{6}, g \cdot \underline{2}=\underline{2}+\gamma \underline{5}, g \cdot \underline{3}=\underline{3}+\xi \underline{4}, \\
& g \cdot \underline{6}=\underline{6}+\beta \underline{1}, g \cdot \underline{5}=\underline{5}+\delta \underline{2}, g \cdot \underline{4}=\underline{4}+\eta \underline{3} .
\end{aligned}
$$

Then

$$
g \cdot f=\left\{\begin{array}{l}
(1-\beta \gamma+\beta \zeta-\gamma \zeta+\beta \lambda) \underline{145}+ \\
(\beta-\delta-\beta \delta \zeta+\zeta-\beta \lambda \delta) \underline{124}+ \\
(\eta-\beta \gamma \eta+\beta-\gamma+\beta \lambda) \underline{135}+ \\
(-\delta \eta+\beta \eta-\beta \delta+1-\beta \delta \lambda \eta) \underline{123}+ \\
(\alpha-\gamma+\zeta-\zeta \gamma \alpha+\lambda) \underline{456}+ \\
(-\alpha \delta+1-\zeta \delta-\alpha \zeta-\lambda \delta) \underline{246}+ \\
(1+\alpha \eta-\gamma \eta-\alpha \gamma+\lambda \eta) \underline{356}+ \\
(\eta-\delta-\alpha \eta \delta+\alpha-\lambda \eta \delta) \underline{236} .
\end{array}\right.
$$

First of all, assume that $F$ has odd characteristic. Putting $\eta=\delta=\alpha=0$ and $\beta=\gamma=-\xi=\frac{1}{2} \lambda$, we get the form $g \cdot f=\left(1+\frac{1}{4} \lambda^{2}\right) \underline{145}+\underline{123}+\underline{246}+\underline{356}$. If $\lambda^{2}+4=0$, then the form is equivalent to $f_{4}$. Otherwise, rescaling 1 reduces the proof to the case where $\lambda=0$. Thus we may assume $f=\underline{145}+\underline{246}+\underline{356}+\underline{123}$. Now, applying the transformation $g$ of (10) again, but now with $\bar{\beta}=\delta=\eta \overline{=}=1$ and $\alpha=\gamma=\zeta=-1$, we get by (11) that $g \cdot f=2(135+246)$, so that $f$ is equivalent to $f_{3}$. This settles the case of odd characteristic. From now on in this proof, assume that $F$ has even characteristic. Take $\psi \in F^{*}$ such that $\psi^{2}+\lambda \psi+1=0$, and consider the transformation $g$ of (10), but now with $\alpha=\delta=\eta=0$ and $\beta=\gamma=\zeta=\psi$. Then $g \cdot f=\underline{123}+\underline{246}+\underline{356}+\lambda \underline{456}$. Thus, $f$ is equivalent to $f_{4}$ if $\lambda=0$, and equivalent to $f_{3}$ otherwise according to Lemma 4.4 . 
4.6. Lemma. If $\mathrm{rk} f=6$, then $f$ is equivalent to either $f_{3}$ or $f_{4}$.

Proof. Let $e_{1}, \ldots, e_{6}$ be a basis of a complement $D$ of $\operatorname{ker} f$ such that (9) is satisfied for $\left.f\right|_{D}$. First, suppose that $f\left(e_{4}, e_{5}, e_{6}\right) \neq 0$. Then, up to rescaling $\underline{6}$, we have

$$
f=\underline{123}+\underline{456}+\sum_{i=1}^{3}\left(\alpha_{i} \underline{i 45}+\beta_{i} \underline{i 46}+\gamma_{i} \underline{i 56}\right) \text { for } \alpha_{i}, \beta_{i}, \gamma_{i} \in F .
$$

Applying a suitable transformation in $E$ preserving the subspace of $E^{*}$ spanned by $\underline{1}, \underline{2}, \underline{3}$ and the subspace spanned by 4,5 , 6 , we can easily reduce study to the case where $f=\underline{123}+\underline{456}+\alpha \underline{145}+\overrightarrow{\beta 2} \overline{46} \mp \gamma 356$ for $\alpha, \beta, \gamma \in F$. If at least two of $\alpha, \beta, \gamma$ are zero, we are done. Without loss of generality, suppose $\beta \gamma \neq 0$. After rescaling by $g \in G$ with $g \cdot \underline{4}=\beta 4, g \cdot 5=\gamma \underline{5}$ and $g \cdot \underline{\sigma}=\beta^{-1} \gamma^{-1} \underline{6}$, we may assume that $f=\underline{123}+456+\bar{\lambda} 145 \overline{+} \underline{356}+\underline{246}$ for some $\lambda \in F$. If $\lambda=0$, then $f$ is equivalent to $f_{3}$ by Lemma 4.4, and if $\lambda \neq 0$, the desired result follows from Lemma 4.5 after rescaling of 1 by $\lambda^{-1}$. Next, suppose that $f\left(e_{4}, e_{5}, e_{6}\right)=0$. Since $\mathrm{rk} f=6$, without loss of generality we may assume that $f\left(e_{1}, e_{4}, e_{5}\right)=1$. Replacing $e_{2}, e_{3}$ by a basis of the kernel of the linear functional $x \mapsto f\left(x, e_{4}, e_{5}\right)$ on $\left\langle e_{1}, e_{2}, e_{3}\right\rangle$, if necessary, we get $f\left(e_{2}, e_{4}, e_{5}\right)=f\left(e_{3}, e_{4}, e_{5}\right)=0$. Replacing $e_{6}$ by $e_{6}+f\left(e_{1}, e_{5}, e_{6}\right) e_{4}-f\left(e_{1}, e_{4}, e_{6}\right) e_{5}$, we also have $f\left(e_{1}, e_{5}, e_{6}\right)=f\left(e_{1}, e_{4}, e_{6}\right)=0$. Thus we are left with the case where $f=\underline{123}+\underline{145}+\alpha \underline{346}+\beta 356+$


nonzero. Thanks to symmetry, we only need consider $\beta \neq 0$. After rescaling, $\underline{\sigma}$, we may put $\beta=1$. Let $g_{1} \in G$ be such that $f \underline{3}=\underline{3}-\delta \underline{2}, g_{1} \underline{5}=\underline{5}-\alpha \underline{4}$ and $g_{1} \underline{i}=\underline{i}$ for $i \neq 3,5$. Then

$$
g \cdot f=\underline{123}+\underline{145}+\underline{356}+(\gamma-\alpha \delta) \underline{246} .
$$

Thus, either $\gamma=\alpha \delta$ and $f$ is equivalent to $f_{4}$, or $\gamma \neq \alpha \delta$ and after rescaling 2 , we arrive at the form discussed in Lemma 4.5 (with $\lambda=0$ ). Hence $f$ is equivalent to $f_{3}$ or $f_{4}$ in all cases.

If $f$ and $f^{\prime}$ are forms on $E$ and $D, D^{\prime}$ are linear subspaces of $E$, then $f \mid D$ is said to be equivalent to $f^{\prime}$ on $D^{\prime}$, if there is $g \in G=G L(E)$ with $g(D)=D^{\prime}$ and $(g f)\left|D^{\prime}=f^{\prime}\right| D^{\prime}$. Furthermore, given the standard basis $e_{1}, \ldots, e_{7}$ of $E$, we shall write $W_{i}$ for the subspace spanned by $\left\{e_{j} \mid j \neq i\right\}$.

4.7. Lemma. If $\mathrm{rk} f=7$ and $E$ has a 6-dimensional subspace $W$ such that $f \mid W$ is equivalent to $f_{3}$ on $W_{7}$, then $f$ is equivalent to one of $f_{5}, f_{7}, f_{9}$.

Proof. Let $e_{1}, \ldots, e_{6}$ be a basis of $W$ such that $f|W=(123+456)| W$. Choose $e_{7} \in E$ such that $f\left(e_{i}, e_{j}, e_{7}\right)=0$ whenever $i, j$ satisfy $1 \leqslant i, j \leqslant 3$ or $4 \leqslant i, j \leqslant 6$ (cf. Lemma 4.1). Then

$$
f=\underline{123}+\underline{456}+\sum_{i=1}^{3} \sum_{j=4}^{6} \alpha_{i j} \underline{i j 7} \text { for some } \alpha_{i j} \in F .
$$


By use of a linear transformation stabilizing the linear subspaces of $E$ spanned by $\underline{1}, \underline{2}, \underline{3}$, by $\underline{4}, \underline{5}, \underline{6}$, and by $\underline{7}$ respectively, we can restrict attention to the case where

$$
f=\underline{123}+\underline{456}+\underline{367}+\alpha \underline{257}+\beta \underline{147} \text { for some } \alpha, \beta \in F .
$$

If $\alpha=\beta=0$, then $f$ is equivalent to $f_{5}$. Without harming generality, assume $\beta \neq 0$. Rescaling $\underline{1}$ and 2 by $\beta^{-1}$ and $\beta$ respectively, we may take $\beta=1$. If $\alpha=0$, then $f$ is equivalent to $f_{7}$. If $\alpha \neq 0$, then choose $\gamma \in F$ with $\gamma^{3}=\alpha$ and rescale $1,2,3,7$ by $\gamma, \gamma^{-2}, \gamma, \gamma^{-1}$, respectively. The resulting form is $f_{9}$. This proves the lemma.

4.8. Lemma. Suppose $\mathrm{rk} f=7$ and $E$ has no 6-dimensional subspace $U$ such that $\left.f\right|_{U}$ is equivalent to $f_{3}$ on $W_{7}$. If there is a subspace $W$ such that $\left.f\right|_{W}$ is equivalent to $f_{4}$ on $W_{7}$, then $f$ is equivalent to $f_{6}$.

Proof. Let $W$ be as stated. There is a basis $e_{1}, \ldots, e_{6}$ of $W$ such that $\left.f\right|_{W}=\underline{125}+\underline{134}+\underline{236}$. Choose $e_{7} \in E$ such that $f\left(e_{i}, e_{j}, e_{7}\right)=0$ for $i, j, 1,2,3$. Then

$$
f=\underline{125}+\underline{134}+\underline{236}+\sum_{i=1}^{6} \sum_{j=4}^{6} \alpha_{i j} i \underline{7} 7 \text { for some } \alpha_{i j} \in F .
$$

Replacing $f$ by $g \cdot f$, where $g \in G$ is given by $g \cdot \underline{1}=\underline{1}-\alpha_{25} \underline{7}, g \cdot \underline{2}=\underline{2}-\alpha_{36}$, $g \cdot \underline{3}=\underline{3}+\alpha_{26} \underline{7}$, and $g \cdot \underline{i}=\underline{i}$ for $i=4,5,6,7$ we have that $\alpha_{25}=\alpha_{26} \equiv \alpha_{36}=\overline{0}$. First of all, assume that $\alpha_{15} \neq 0$. Then $\alpha_{16} \neq 0$, because otherwise $\left.f\right|_{W_{4}}=$ $\left(125+236+\alpha_{15} 157+\alpha_{35} \underline{357}+\alpha_{56} \underline{567)} \mid W_{4}\right.$ would be equivalent to $f_{3}$ on $\overline{W_{7}}$. (Take $h \in G L \overline{\left(W_{4}\right)}$ with $\overline{h .1}=1-\alpha_{35} \alpha_{15}{ }^{4} 3, h \cdot 6=6-\alpha_{35} \alpha_{15}^{-1} \underline{5}$, and $h \cdot \underline{i}=\underline{i}$ for $i=1,2,3,5,7$, and apply the 6-dimensional analogue of Lemma 4.4 to $\left.h \cdot\left(f \mid \bar{W}_{4}\right)\right)$.

We claim that $\alpha_{56}=0$. For if $\alpha_{56} \neq 0$, then $g_{1} \in G$ with $g_{1} \cdot \underline{1}=\underline{I}-\alpha_{56} \alpha_{16}^{-1} \underline{5}$, $g_{1} \cdot \underline{4}=\underline{4}-\alpha_{35} \alpha_{56}^{-1} \alpha_{16} \underline{7}$, and $g_{1} \cdot \underline{i}=\underline{i}$ for $i \neq 1,4$ applied to $\bar{f}$ yields

$$
\left.\left(g_{1} \cdot f\right)\right|_{W_{4}}=\left(\underline{125}+\underline{236}+\alpha_{15} \underline{157}+\alpha_{16} \underline{167}+\gamma \underline{137}\right) \mid W_{4}
$$

for some $\gamma \in F$. This implies by Lemma 4.4 that $f \mid g^{-1} W_{4}$ is equivalent to $f_{3}$ on $W_{7}$, contradictory to the assumption. Thus, $f=\underline{125}+\underline{236}+$ $\alpha_{35} \underline{357}+\alpha_{16} \underline{167}+\alpha_{15} \underline{157}+\underline{134}+\sum_{i=1}^{6} \delta_{i} i 47$, for certain $\delta_{i} \in F$. Consider $f \mid W_{4}$, once again. In view of Lemma 4.4 and the assumption, we have $\alpha_{35} \neq \dot{0}$, and in view of Lemma 4.5, we have $\alpha_{15}^{2}=4 \alpha_{35} \alpha_{16}$. In particular, $F$ has odd characteristic and $\alpha_{15} \neq 0$. Applying $g_{2} \in G$ with $g_{2} \cdot \underline{1}=\underline{1}-\frac{1}{2} \alpha_{15} \alpha_{16}^{-1} \underline{3}$, $g_{2} \cdot \underline{2}=\underline{2}+\frac{1}{2} \alpha_{15} \underline{7}, g_{2} \cdot \underline{6}=\underline{6}-\frac{1}{2} \alpha_{15} \alpha_{16}^{-1} \underline{5}, g_{2} \cdot \underline{7}=\alpha_{16}^{-1} \underline{7}$, and $g_{2} \cdot \underline{i}=\underline{i}$ for $i \neq 1,2,6,7$ to $f$ leads to the form

$$
f^{\prime}=g_{2} \cdot f=\underline{125}+\underline{236}++\underline{134}+\underline{167}+\sum_{i=1}^{6} \delta_{i} \underline{i 47}, \text { with } \delta_{i} \in F .
$$

Now, consider $\left.\left(f^{\prime}\right)\right|_{W_{1}}=\underline{(236}+\left(\sum_{i=2}^{6} \delta_{i} \underline{i} \underline{47}\right) \mid W_{1}$. 
Clearly, this form is equivalent to $f_{3}$ on $W_{7}$ if it is nondegenerate on $W_{1}$. Hence $\delta_{5}=0$. Next, consider

$$
\begin{aligned}
\left(f^{\prime}\right) \mid W_{6}= & \left.\frac{(125}{\delta_{3}(\underline{3}}+\underline{1}\left(\underline{3}+\delta_{1} \underline{4}\right) \underline{4}(\underline{4}+\underline{4}+\underline{7})\right) \mid W_{6} .
\end{aligned}
$$

By Lemma 4.4 , this form is equivalent to $f_{3}$ on $W_{7}$, unless $\delta_{3}=0$. Hence, $\delta_{3}=0$. Consider $\left.\left(f^{\prime}\right)\right|_{W_{3}}=\left.\left(125+\left(\underline{6}+\delta_{1} \underline{4}\right) \underline{71}+\delta_{2} 247+\delta_{6}\left(\underline{6}+\delta_{1} \underline{4}\right) \underline{47}\right)\right|_{W_{3}}$. Again, by Lemma 4.4 and the assumption, we get $\delta_{6}=\overline{0}$. Consider $\left.f^{\prime}\right|_{w_{5}}=$ $\left(236+134+167+\delta_{1} 147+\delta_{2} 247\right) \mid W_{5}$. If $\delta_{2}=0$, then $\delta_{1}=0$ by Lemma 4.5; if $\delta_{1}=0$, then $\delta_{2}=0$ by Lemma 4.4. But $\delta_{1}=\delta_{2}=0$ yields $f^{\prime}=125+236+$ $134+167$, which is equivalent to $f_{6}$. Thus, the case where $\delta_{1}, \delta_{2} \neq \overline{0 \text { remains. But }}$ then, according to Lemma 4.5 , we must have $\delta_{1}^{2}=-4 \delta_{2}$. Applying $g_{3} \in G$ given by $g_{3} \cdot \underline{2}=\underline{2}+2 \delta_{1}^{-1} 1, g_{3} \cdot \underline{3}=3-2 \delta_{1}^{-1} \underline{7}, g_{3} \cdot \underline{4}=\underline{4}-2 \delta_{1}^{-1} \underline{6}, g_{3} \cdot \underline{7}=-4 \delta_{1}^{-2} \underline{7}$ and $g_{3} \cdot \underline{i}=\underline{i}$ for $i=1,5,6$ to $f^{\prime}$, we obtain $g_{3} \cdot \bar{f}^{\prime}=\underline{125}+134+236+247$, which is clearly equivalent to $f_{6}$. This settles the case where $\alpha_{15} \neq 0$.

Thus, for the rest of the proof, we may assume $f=\underline{125}+\underline{134}+$ $\underline{236}+\sum_{i=1}^{6} \sum_{j=4}^{6} \alpha_{i j} i j 7$, with $\alpha_{15}=\alpha_{25}=\alpha_{26}=\alpha_{36}=0$.

Suppose that $\alpha_{14} \neq 0$. If $\alpha_{34}=0$, then a permutation of basis elements (according to $(3,1,2)(4,5,6)$ on the indices) reduces this case to the previous one. If $\alpha_{34} \neq 0$, then apply $g_{4} \in G$ with $g_{4} \cdot \underline{I}=1-\alpha_{34} 7$ and $g \cdot \underline{i}=i$ for $i \neq 1$ to $f$ so as to obtain $g_{4} \cdot f=f-\alpha_{34} 347-\alpha_{34} 257$; the permutation $(4, \overline{5})(2,3)$ on the basis elements then leads $g_{4} \cdot f$ to a form with $\overline{\alpha_{15}} \neq 0$ and $\alpha_{25}=\alpha_{26}=\alpha_{36}=0$, a situation that has been dealt with before. Thus we may assume $\alpha_{14}=0$. Similarly, we may assume $\alpha_{34}=0$. Now,

$$
\begin{aligned}
& f= \underline{125}+\underline{134}+\underline{236}+\alpha_{16} \underline{167}+\alpha_{24} \underline{247}+ \\
& \alpha_{35} \underline{357}+\alpha_{45} \underline{457}+\alpha_{46} \underline{467}+\alpha_{56} \underline{567}
\end{aligned}
$$

If $\alpha_{46} \neq 0$, then $g_{5} \in G$ given by $g_{5} \cdot \underline{1}=\underline{1}-\alpha_{56} \alpha_{46}^{-1} \frac{5}{5}, g_{5} \cdot \underline{2}=\underline{2}+$ $\alpha_{56} \alpha_{24} \alpha_{46}^{-1} \underline{7}, g_{5} \cdot \underline{4}=\underline{4}-\alpha_{45} \alpha_{46}^{-1} \underline{5}, g_{5} \cdot \underline{6}=\underline{6}-\alpha_{45} \alpha_{16} \alpha_{46}^{-1} \underline{7}, g_{5} \underline{i}=\bar{i}$ for $i=3,5,7$, is such that

$$
\left.\left(g_{5} f\right)\right|_{W_{3}}=\left.\left(\underline{125}+\alpha_{16} \underline{167}+\alpha_{24} \underline{247}+\alpha_{46} \underline{467}\right)\right|_{W_{3}}
$$

is equivalent to $f_{3}$ on $W_{7}$, cf. Lemma 4.4 . Hence, $\alpha_{46}=0$, and by symmetry, also, $\alpha_{56}=0$. Consequently,

$$
f=\underline{125}+\underline{134}+\underline{236}+\alpha_{16} \underline{167}+\alpha_{24} \underline{247}+\alpha_{35} \underline{357} .
$$

Since $\mathrm{rk} f=7$, at least one of $\alpha_{16}, \alpha_{24}, \alpha_{35}$ is nonzero. If exactly one of them is nonzero, we are done. Suppose therefore, that at least two of them, say $\alpha_{16}$ and $\alpha_{24}$ (without loss of generality) are nonzero. Suppose that $F$ has even characteristic. Then $g_{6} \in G$ determined by $g_{6} \underline{\underline{1}}=\underline{1}+\alpha_{2} \mu \underline{\underline{2}}, g \cdot \underline{4}=\underline{4}+\alpha_{1} \mu \underline{6}, g_{6} \cdot \underline{7}=$ $\underline{7}+\mu \underline{3}, \quad$ and $g \cdot \underline{i}=\underline{i}$ for $i \equiv 2,3,5, \overline{6}$, where $\bar{\mu} \in F^{\bar{x}}$ is such that 


$$
\begin{aligned}
\mu^{2}+\mu+ & \left(\alpha_{1} \alpha_{2}\right)^{-1}=0, \text { satisfies } \\
& \left.\left(g_{6} f\right)\right|_{W_{5}}=\left(\underline{134}+\alpha_{1} \underline{167}+\alpha_{2} \underline{247}+\alpha_{2} \underline{\mu 234}\right) \mid W_{5} .
\end{aligned}
$$

The latter form is equivalent to $f_{3}$ on $W_{7}$ by Lemma 4.4. This contradiction shows that $F$ has odd characteristic. But then $\left.f\right|_{5}$ is equivalent to $f_{3}$ on $W_{7}$ by Lemma 4.5, leading to the final contradiction. This ends the proof of Lemma 4.8.

4.9. Lemma. Suppose $\mathrm{rk} f=7$ and $E$ has no 6-dimensional subspace $U$ such that $f \mid u$ has rank 6 . Then $f$ is equivalent to $f_{8}$.

Proof. In view of the assumption, there must be a 5-dimensional subspace $W$ of $E$ such that $f \mid W$ is equivalent to $f_{2}$ on $\left\langle e_{1}, \ldots, e_{5}\right\rangle$. Take such a subspace and let $e_{1}, . ., e_{7}$ be a basis of $E$ such that $W=W_{6} \cap W_{7}$ and $f|W=(123+145)| W$. Now, $f\left(e_{6}, e_{i}, e_{j}\right)=f\left(e_{7}, e_{i}, e_{j}\right)=0$ for $i, j=1, \ldots, 5$, for otherwise $E$ has a 6dimensional subspace $V$ on which $f \mid V$ has rank 6 . If $f\left(e_{2}, e_{6}, e_{7}\right) \neq 0$, then $f \mid W_{3}$ clearly has rank 6 , a contradiction. Hence, $f\left(e_{2}, e_{6}, e_{7}\right)=0$, and similarly, $f\left(e_{3}, e_{6}, e_{7}\right)=f\left(e_{4}, e_{6}, e_{7}\right)=f\left(e_{5}, e_{6}, e_{7}\right)=0$. Since $\mathrm{rk} f=7$, we must have $f\left(e_{1}, e_{6}, e_{7}\right) \neq 0$, and $f$ is equivalent to $f_{8}$.

Now, Lemmas $4.7,4.8$, and 4.9 classify all forms $f \in \mathrm{Alt}_{3}(E)$ with $\mathrm{rk} f=7$. Thus, together with Lemmas 4.2, 4.3, and 4.6, this completes the proof of Theorem 2.1.

\section{The Classification over Perfect Fields of Cohomological Dimension at most 1.}

Let $E$ be a vector space of dimension $n<\infty$ over a perfect field $F$, and let $\bar{F}$ be the algebraic closure of $F$. Galois cohomology provides a tool to keep track of the inequivalent forms for $E$ which become equivalent when extended to forms $\bar{f}$ on $\bar{E}=E \otimes_{F} \bar{F}$. We shall first recall some basic notions from SERRE [8].

5.1. Definitions. For $L$ a Galois field over $F$ (within $\bar{F}$ ), denote by $\Gamma_{L}$ the Galois group of $\bar{F}$ over $L$, and set $\Gamma=\Gamma_{F}$. The latter is a profinite group, i.e., it is compact and discontinuous when topologized in such a way that the groups $\Gamma_{L}$ for $L$ an extension field of $F$ (contained in $\bar{F}$ ) form the collection of open subgroups of $\Gamma$. Set $\bar{G}=G L(\bar{E})$ and suppose $A$ is a subgroup of $G$ topologized by the discrete topology. Then $\Gamma$ has a natural action from the right on $A$ given by

$$
a^{\sigma}=\left(a_{i j}^{\sigma}\right)_{1 \leqslant i, j \leqslant n} \text { for } a=\left(a_{i j}\right)_{1 \leqslant i, j \leqslant n} \in A \text { and } \sigma \in \Gamma \text {. }
$$

A cocycle of $\Gamma$ with values in $A$ is a continuous map $\Gamma \rightarrow A$, denoted by $\sigma \mapsto a_{0}$ $(\sigma \in \Gamma)$, such that $a_{\sigma \tau}=a_{\sigma}^{\tau} a_{\tau}$ for any $\sigma, \tau \in \Gamma$. Two such cocycles $a$ and $a^{\prime}$ are called cohomologous if there is $b \in A$ with $a_{\sigma}^{\prime}=b^{\sigma} a_{\sigma} b^{-1}$ for every $\sigma \in \Gamma$. This defines an equivalence relation on the set of all cocycles of $\Gamma$ with values in $A$. The set of equivalence classes is called the first cohomology of $\Gamma$ in $A$ and denoted by $H^{\mathrm{l}}(\Gamma, A)$. If $A$ is abelian, $H^{\mathrm{l}}(\Gamma, A)$ can be given a group structure and higher cohomology groups $H^{i}(\Gamma, A)$ can be defined. Of course, there is always a distinguished element $1 \in H^{1}(\Gamma, A)$, namely the class corresponding to the trivial cocycle $\sigma \mapsto 1$ $(\sigma \in \Gamma)$. We recall that a perfect field $F$ is said to have cohomological dimension at 
most 1 if the cohomological dimension of the Galois group $\Gamma$ is at most 1. (This means that for each prime $p$ and each discrete simple $\Gamma$-module $A$ with $p A=0$, we have $H^{2}(\Gamma, A)=0$.) The reader is referred to SERRE [8] for further details. Here, we just mention that finite fields have cohomological dimension at most 1 .

Suppose $f \in \operatorname{Alt}_{3}(E)$. We are interested in the set $T(F, f)$ of equivalence classes of forms $f^{\prime} \in \operatorname{Alt}_{3}(E)$ such that $\bar{f}^{\prime}$ and $\bar{f}$ are equivalent forms in $\operatorname{Alt}_{3}(\bar{E})$.

5.2. Theorem. Let $F$ be a perfect field, and suppose $f \in \operatorname{Alt}_{3}(E)$. Retain the above notation, and put $A=\widetilde{G}_{f}$. Then the following holds:

(i) There is a bijective map $\theta: T(F, f) \rightarrow H^{1}(\Gamma, A)$, given by $\theta\left(f^{\prime}\right) \sigma=g^{\sigma} g^{-1}$ $\left(f^{\prime} \in T(F, f), \sigma \in \Gamma\right)$, where $g \in \bar{G}$ is such that $g \cdot f^{\prime}=f$.

(ii) If $F$ has cohomological dimension at most 1 , and if $A^{0}$ denotes the connected component of unity in $A$, viewed as a linear algebraic group over $\bar{F}$, then $A^{0}$ is a normal subgroup of $A$ preserved by $\Gamma$, and the natural map $H^{1}(\Gamma, A) \rightarrow H^{1}\left(\Gamma, A / A^{0}\right)$ is bijective.

Proof. (i) See Serre [8] Chapter III, Proposition 1.

(ii) See SERRE [8] Chapter III, Theorem 1, Conjecture 1, Supplement 2, and Corollary 3.

We are now ready for the

5.3. Proof of Theorem 2.2. Suppose $f \in \operatorname{Alt}_{3}(E), f \neq 0$, and set $A=\overline{G_{f}}$.

If $\bar{f}$ of the form $\bar{f}_{i}$ for some $i(1 \leqslant i \leqslant 9)$ with $i \neq 3,5,9$, then $A$ is connected, cf. Table 1 , so $f$ is equivalent to $f_{i}$ by the above theorem.

Suppose $\bar{f}=\bar{f}_{3}$ (in other words, $G \cdot f \in T\left(F, f_{3}\right)$ ). Then $A / A^{0} \cong \mathbb{Z}_{2}$ by Table 1 and $\Gamma$ acts trivially on $A / A^{0}$. It is easy to see that $H^{1}\left(\Gamma, A / A^{0}\right)$ coincides with the set of continuous group morphisms $\Gamma \rightarrow A / A^{0}$. But any such morphism is related to a subgroup $\Delta$ of $\Gamma$ of index 2, whence to a quadratic extension $K$ of $\Gamma$ (determined by $\Delta=\Gamma_{K}$ ). By Theorem 5.2 and $\S 3.10$, there is a unique quadratic extension $K$ of $F$ (within $\bar{F}$ ) such that $f$ is equivalent to $f_{10, \lambda}$ for each $\lambda \in F$ such that $P_{\lambda}(X)$ is irreducible in $F[X]$ and reducible in $K[X]$. The same argument holds for $\bar{f}=\bar{f}_{5}$, with $f_{11, \lambda}$ instead of $f_{10, \lambda}$.

Finally, suppose $\bar{f}=\bar{f}_{9}$. If $F$ has characteristic 3 , then $A$ is connected, as $\mu_{3}=\{1\}$, so $f$ is equivalent to $f_{9}$ by Theorem 5.2. Suppose from now on that the characteristic of $F$ is distinct from 3. If $A / A^{0}=\{1\}$, there is nothing to prove. So, suppose $A / A^{0} \neq\{1\}$. Then, by Table 1 , we get $A / A^{0} \cong \mathbb{Z}_{3} \cong \mu_{3}$ and there is $\sigma \in \Gamma$ acting on $A / A^{0}$ as it does on $\mu_{3} \subseteq \bar{F}$. Write $\mu_{3}=\{1, \omega, \bar{\omega}\}$.

Assume, first of all, that $\mu_{3} \subseteq F$. Then the action of $\Gamma$ on $\mu_{3}$ is trivial, so a cocycle is a continuous group morphism $\Gamma \rightarrow \mu_{3}$ whose kernel is a normal subgroup of $\Gamma$ of index 1 or 3 . Of course, index 1 only occurs in the case of the trivial cocycle. Thus, any nontrivial cocycle has a kernel $\Delta$ of index 3 in $\Gamma$ corresponding to a Galois extension of $F$ of degree 3 with group $\Gamma / \Delta$. Applying this to the cocycle $\theta(f)$ of Theorem 5.2, this means that for $f$ there is a unique cubic Galois extension $K$ of $F$ 
such that $f$ is $K$-equivalent to $f_{9}$. Let $\gamma \in \Gamma$ be such that $\Gamma=\Delta \cup \Delta \gamma \cup L$ There are two cocycles in $H^{1}(\Gamma, A)$ whose kernel is $\Delta$; they are determined by $\omega$ and $\gamma \mapsto \omega^{2}$, respectively. Hence, there are exactly two classes of forms $T\left(F, f_{9}\right)$ which are $K$-equivalent to $f_{9}$ but not equivalent to $f_{9}$. Writing $K=F$ where $\alpha^{3}=\mu \in F$ (see LANG [6] Chapter 8, Theorem 10), we can represent tr by $f_{12, \mu}$ and $f_{12, \mu^{2}}$, respectively (cf. $\S 3.12$ ).

Suppose, next, that $\mu_{3}$ is not contained in $F$. Let $X$ be the kernel of the action c on $\mu_{3}$. Then $X$ has index 2 in $\Gamma$. Given a cocycle $\sigma \mapsto a_{\sigma}(\sigma \in \Gamma)$, call the gr $\Delta_{a}=\left\{\sigma \in \Gamma \mid a_{\sigma}=1\right\}$ the kernel of the cocycle. Clearly, $\Delta_{a}$ has index 3 in $\Gamma$, un it is the kernel of the trivial cocycle. Suppose that $\Delta_{a}$ has index 3 in $\Gamma$. $T$ $\Delta_{a} \cap X$ is a subgroup of $X$ of index 3, which is normal in $X$ since it is the ke: of the group morphism $\gamma \mapsto a_{\gamma}$ from $X$ to $\mu_{3}$. Thus, there is $\sigma \in X$ such that partitioned by $\left(\Delta_{a} \cap X\right) \sigma^{i}$ for $i=0,1,2$, whence $\Gamma=\Delta_{a} \cup \Delta_{a} \sigma \cup \Delta_{a} \sigma^{2}$. N $\Delta_{a} \cap X$ has index 2 in $\Delta_{a}$, and we have $\tau \in \Delta_{a}$ such that $\omega^{\tau}=\bar{\omega}=\omega^{2}$ and $\Delta_{a}$ $\left(\Delta_{a} \cap X\right) \cup\left(\Delta_{a} \cap X\right) \tau$. Moreover, the cocycles $\gamma \mapsto a_{\gamma} \omega^{\gamma} \omega^{-1}$ and $\gamma \mapsto a_{\gamma} \bar{\omega}^{\gamma} \bar{c}$ are cohomologous to $a$ and have kernels $\Delta_{a} \cap X\left\{1, \tau \sigma^{2}\right\}$ and $\Delta_{a} \cap X\{1$, respectively. Since these kernels are open subgroups of $\Gamma$, it follows $(\tau \sigma)^{2} \in \Delta_{a} \cap X$. Furthermore, $\Delta_{a} \cap X=\cap_{c}\left\{\gamma \in \Gamma \mid c_{\gamma}=1\right\}$, where $c$ runs c all cocycles cohomologous to $a$, is a normal subgroup of $\Gamma$, so $\Gamma /\left(\Delta_{a} \cap X\right) \cong$ the symmetric group on 3 letters. Writing $K$ for the extension of $F$ correspondin $\Delta_{\theta(f)}$, we obtain that $K(\omega)$ is Galois over $F$ with group $\left\langle\left.\sigma\right|_{K(\omega)},\left.\tau\right|_{K(\omega)}\right\rangle$ $S_{3}$, that $f$ is $K(\omega)$-equivalent to $f_{9}$ and that the $K(\omega)$-equivalence class contair $f_{9}$ consists of exactly three equivalence classes of forms on $E$. (For, there are ex ly three classes of cocycles $b$ with $\Delta_{b} \cap X=\Delta_{\theta(f)} \cap X$, cf. Theorem 5.2.) By 3 the forms $f_{9}, \lambda f_{9}, \lambda^{2} f_{9}$, where $\lambda \in F(\omega)$ is such that $X^{3}-\lambda$ is irreducible , $F(\omega)$ but reducible over $K(\omega)$, are mutually $F(\omega)$-inequivalent, and $K(\omega)$-equiva to $f_{9}$. Let $\alpha \in K$ satisfy $\alpha^{3}=\lambda$. Then $\alpha^{\tau} \alpha^{-1}$ is fixed by $\sigma$ (for $\alpha^{\sigma}=\alpha \omega^{i}$ for si $i \in\{1,2\}$, and $\alpha^{\tau \sigma}=\alpha^{\sigma^{-1} \tau}=\alpha^{\tau} \omega^{2 i}$ ), whence $\alpha^{\tau} \alpha^{-1} \in F(\omega)$. By Hilbert 90, LANG [6], there is $\beta \in F(\omega)$ such that $\beta^{r} \beta^{-1}=\alpha^{\tau} \alpha^{-1}$. Set $\mu=\lambda \beta^{-3}$. Thi an element of $F$. As for $i=0,1,2$ the forms $\mu^{i} f_{9}$ are clearly $F(\omega)$-equivalen $\lambda^{i} f_{9}$, they are mutually inequivalent forms on $E$ which are $K(\omega)$-equivalent to Since $f$ is $K(\omega)$-equivalent to $f_{9}$, it follows that $f$ is, up to equivalence, one of $\mu^{i} f_{9}=f_{12, \mu^{i}}(i=1,2)$. Also from the above we have that for $\lambda, \mu \in F$ the elen $\lambda^{-1} \mu$ has a cube root in $F(\omega)$ if and only if $f_{9}$ is equivalent to $\lambda^{-1} \mu f_{9}$, which 0 ously amounts to the equivalence of $\lambda f_{9}$ and $\mu f_{9}$. This establishes Theorem 2.2.

5.4. Concluding remark. It is not hard to generalize part of Theorem 2.2 to case of an arbitrary perfect field $F$ by use of SERRE [8]. For, by Proposition 6, ] position 3 and Lemma 1 of Chapter III [loc. cit.], we have $H^{1}(\Gamma, U)=0$ wheneve is one of the groups $(\bar{F})_{*}^{m}, S p(2 m, \bar{F}), G L(m, \bar{F})(m \in \mathbb{N})$, and using the exact quences of Propositions 6 and 8 of Chapter I [loc. cit.], we get $H^{1}(\Gamma, S L(m, \bar{F}))$ so that

$$
\begin{aligned}
& H^{1}\left(\Gamma, G_{i}\right)=0 \text { if } i=1,2,4,6,8 \text { and } \\
& H^{1}\left(\Gamma, G_{i}\right) \cong H^{1}\left(\Gamma, \mathbb{Z}_{2}\right) \text { if } i=3,5 .
\end{aligned}
$$


This implies that for $i=1,2,3,4,5,6,8$, any trilinear form which is $\bar{F}$-equivalent to $f_{i}$ must be equivalent to either $f_{i}$ or one of $f_{j, \lambda}$ (where $j=10,11$, if $i=3,5$, respectively).

Acknowledgement. We are grateful to Professor T.A. Springer for a helpful discussion.

\section{References}

[1] Borel, A., Linear Algebraic Groups, Benjamin, New York, 1969.

[2] Bourbaki, N., Elements de Mathématique Algèbre, Chapitres 1-3, Hermann, Paris, 1971.

[3] Cresp, J., Orbits in $\bigwedge_{i}^{3} k^{7}$, Kummer manifolds and the cohomology of a hyperplane section of a Grassmannian, Thesis,1976, Newcastle upon Tyne.

[4] Dieudonné, J., La géométrie des groupes classiques, Springer, Berlin, 1955.

[5] Gurevich, G.B., Foundations of the Theory of Algebraic Invariants, Noordhoff, Groningen, 1964.

[6] Lang, S., Algebra, Addison-Wesley, Reading MA, 1966.

[7] Schouten, J.A., Klassifizierung der alternierenden Grossen dritten Grades in 7 Dimensionen, Rend. Circ. Matem. Palermo, 55 (1931) 137-156.

[8] Serre, J.-P., Cohomologie Galoisienne, Lecture Notes in Math., 5, Springer, Berlin, 1964.

[9] Springer, T.A., Oktaven, Jordan-Algebren und Ausnahmegruppen, Lecture Notes, Gottingen, 1963.

[10] Migliore, The determination of the maximal subgroups of $G_{2}(q) q$ odd, Phd. Thesis, University of California at Santa Cruz, 1982. 